\title{
A specific phosphorylation regulates the protective role of $\alpha A$-crystallin in diabetes
}

\author{
Anne Ruebsam, ' Jennifer E. Dulle, ${ }^{1}$ Angela M. Myers, ${ }^{1}$ Dhananjay Sakrikar, ${ }^{2}$ Katelyn M. Green, ${ }^{1}$ \\ Naheed W. Khan, ${ }^{1}$ Kevin Schey, ${ }^{3}$ and Patrice E. Fort ${ }^{1,4}$ \\ 'Department of Ophthalmology and Visual Sciences, University of Michigan, Ann Arbor, Michigan, USA. ${ }^{2}$ The Binding \\ Site Inc., Rochester, Minnesota, USA. ${ }^{3}$ Department of Biochemistry and Ophthalmology and Visual Sciences, Vanderbilt \\ University, Nashville, Tennessee, USA. ${ }^{4}$ Department of Molecular and Integrative Physiology, University of Michigan, Ann \\ Arbor, Michigan, USA.
}

\begin{abstract}
Neurodegeneration is a central aspect of the early stages of diabetic retinopathy, the primary ocular complication associated with diabetes. While progress has been made to improve the vascular perturbations associated with diabetic retinopathy, there are still no treatment options to counteract the neuroretinal degeneration associated with diabetes. Our previous work suggested that the molecular chaperones $\alpha$-crystallins could be involved in the pathophysiology of diabetic retinopathy; however, the role and regulation of $\alpha$-crystallins remained unknown. In the present study, we demonstrated the neuroprotective role of $\alpha$ A-crystallin during diabetes and its regulation by its phosphorylation on residue 148 . We further characterized the dual role of $\alpha A-c r y s t a l l i n$ in neurons and glia, its essential role for neuronal survival, and its direct dependence on phosphorylation on this residue. These findings support further evaluation of $\alpha \mathrm{A}$-crystallin as a treatment option to promote neuron survival in diabetic retinopathy and neurodegenerative diseases in general.
\end{abstract}

Conflict of interest: The authors have declared that no conflict of interest exists.

Submitted: October 9, 2017

Accepted: January 17, 2018

Published: February 22, 2018

Reference information: JCI Insight. 2018;3(4):e97919. https:// doi.org/10.1172/jii.insight.97919.

\section{Introduction}

Diabetic retinopathy (DR) is a major complication associated with diabetes that remains the number one cause of vision loss in the working age population (1). Treatment with anti-VEGF agents or corticosteroids benefit a subset of patients with advanced retinopathy, specifically macular edema (2), but more effective treatments are needed for the early stage of the disease. Over the past two decades, numerous studies have demonstrated the neurodegenerative element of DR (3-5), but the underlying mechanisms of neurodegeneration remain to be clearly established.

Several groups, including ours $(6,7)$, have demonstrated that retinal neurodegeneration in animal models of diabetes is consistently associated with increased levels of several members of the crystallin protein family. Crystallin proteins are grouped in 3 major classes: $\alpha-, \beta-$, and $\gamma$-crystallins. While the role of $\gamma$-crystallins remains poorly understood outside of the ocular lens, $\alpha$ - and $\beta$-crystallin proteins have gained increasing interest from the neurodegenerative research community due to the demonstration of their implication in various neurodegenerative diseases (8-10). While $\alpha \mathrm{B}$ - and $\beta \mathrm{A} 1 / \mathrm{A} 3$-crystallins are known to control the integrity of the vasculature in the central nervous system, in part through regulation of VEGF function $(10,11), \alpha \mathrm{A}$ - and $\alpha \mathrm{B}$-crystallins have been implicated in regulating neuronal cell survival in multiple neurodegenerative conditions, including stroke (12), endophthalmitis (13), and uveitis $(14,15), \alpha \mathrm{A}$ - and $\alpha \mathrm{B}$-crystallins are protective in the context of hypoxia (16), while only $\alpha \mathrm{A}$-crystallin demonstrates a protective potential against autoimmune uveitis (14), suggesting specific and independent mechanisms for different crystallins. $\alpha$-Crystallins regulate the unfolded protein response (UPR) or ER stress response, in which chaperone proteins play a key role to prevent protein aggregation and subsequent cell toxicity and cell death. The cellular specificity of this phenomenon and its regulation by $\alpha$-crystallins during neurodegenerative diseases remains unclear.

In addition to our data in animal models $(6,17)$, recent reports have suggested that $\alpha$-crystallins also accumulate in ocular tissues of diabetic patients, supporting an important role for this stress response in the pathology of DR $(18,19)$. We showed that overexpression of $\alpha$-crystallins protects retinal neurons in culture and that diabetes dramatically reduces the solubility and alters the subcellular localization of 


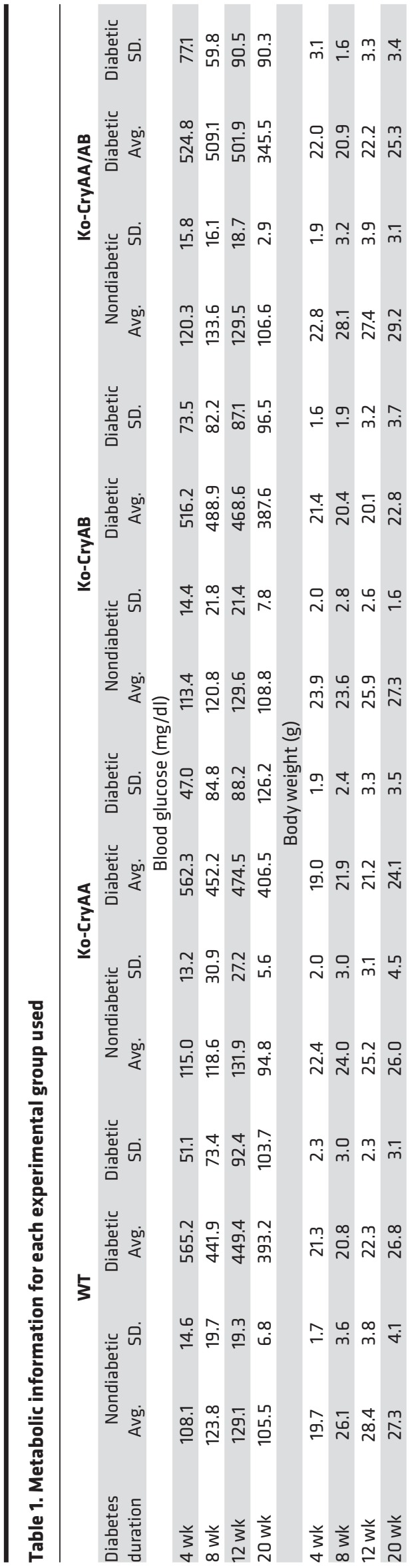

$\alpha$-crystallins (17). Such changes are associated with decreased chaperone activity and protective capacity of $\alpha \mathrm{B}$-crystallin, in part due to posttranslational modifications (PTMs) (20), such as its phosphorylation (21). However the nature and impact of $\alpha \mathrm{A}$-crystallin PTM remains poorly understood. Thus, this study focused on testing the hypothesis that $\alpha$-crystallins play a key role in neuronal survival in the central nervous system, a role regulated by key PTMs that are affected by chronic neurodegenerative conditions.

\section{Results}

Lack of $\alpha A$-crystallin enhances diabetes-associated retinal neurodegeneration. To better understand the role of $\alpha$-crystallin proteins, which accumulate in the retina during diabetes $(6,17)$, we assessed the impact of diabetes in mice lacking expression of $\alpha \mathrm{A}$-crystallin (Ko-CryAA), $\alpha \mathrm{B}$-crystallin (Ko-CryAB), or both (Ko-CryAA/AB). $\alpha \mathrm{A}$ - and $\alpha \mathrm{B}-$ crystallin-KO mice have only been partially characterized previously $(22,23)$, so we analyzed the main tissues in which $\alpha$-crystallins have been reported and found no obvious defects in heart, brain, and retina (Supplemental Figure 1, A-C; supplemental material available online with this article; https://doi. org/10.1172/jci.insight.97919DS1). Other than the cataract formation previously reported in the $\alpha \mathrm{A}$-crystallin-KO mice, the only gross anatomical difference observed was a slight but significant increase in the retinal thickness in the double-KO mice. This increased retinal thickness seems to be primarily due to a "compaction" of the tissue secondary to the cataract formation and relative decreased size of the lens and, as a result, the eye. Indeed, no differences in the overall retinal architecture and network could be detected by histology (Supplemental Figure 1) or immunostaining for specific cell types (data not shown). This is also consistent with the absence of signs of proliferation or apoptosis in nondiabetic animals at any time point. Body weight and blood glucose levels were measured longitudinally throughout the study and confirmed that the absence of crystallin had no effect on the animals' sensitivity to diabetes induction (Tables 1 and 2). Postmortem analysis of the pancreas weight and islet content also confirmed this finding (Supplemental Figure 1D).

$\alpha$-Crystallins are protective in several animal models of retinal neurodegenerative diseases, so we assessed the effect of the lack of $\alpha \mathrm{A}$ - and/or $\alpha \mathrm{B}$-crystallin on retinal cell survival. As previously reported, increased cell death was continuously observed in WT animals starting 8 weeks after diabetes induction. As predicted, $\alpha \mathrm{A}$-crystallin-KO mice consistently had a higher level of retinal cell death, starting after 4 weeks of diabetes (Figure 1A). Unexpectedly though, lack of $\alpha \mathrm{B}$-crystallin was consistently associated with a reduced level of cell death compared with WT diabetic mice. Despite this seemingly protective effect of lack of $\alpha \mathrm{B}$-crystallin, concomitant loss of both $\alpha$-crystallin (double-KO) proteins enhanced diabetes-induced retinal cell death (Figure 1A). While, at this early time point, previous studies have reported apoptotic cells localized away from the vasculature (3), since this method does not discriminate between cell types, a contribution of nonneuronal cells to the cell death rate observed cannot be excluded.

Type 1 diabetes reduces retinal thickness in patients with minimal to no vascular lesions (24). Using optical coherence tomography (OCT), we assessed the retinal thickness and observed thinning of the retina very early in the $\alpha \mathrm{A}$-crystallin-KO mice, as early as 2 weeks after diabetes induction, but not in the WT or the $\alpha \mathrm{B}$-crystallin-KO mice (Figure $1 \mathrm{~B}$ ). Because lack of $\alpha \mathrm{A}$-crystallin is associated with progressive cataract formation, which prohibits performing OCT analysis at later points, we also performed the 
Table 2. Number of animals for each experimental group used

\begin{tabular}{|c|c|c|c|c|c|c|c|c|}
\hline & \multicolumn{2}{|c|}{ WT } & \multicolumn{2}{|c|}{ Ko-CryAA } & \multicolumn{2}{|c|}{ Ko-CryAB } & \multicolumn{2}{|c|}{ Ko-CryAA/AB } \\
\hline & Nondiabetic & Diabetic & Nondiabetic & Diabetic & Nondiabetic & Diabetic & Nondiabetic & Diabetic \\
\hline 4 wk & 18 & 22 & 18 & 22 & 17 & 20 & 20 & 23 \\
\hline $12 \mathrm{wk}$ & 34 & 41 & 16 & 19 & 24 & 17 & 16 & 16 \\
\hline 20 wk & 9 & 22 & 13 & 32 & 9 & 22 & 13 & 24 \\
\hline
\end{tabular}

analysis of retinal thickness by postmortem histologic measurements. This method revealed that past 4 weeks of diabetes, retinal thickness slightly decreased in WT diabetic animals, while being significantly more reduced in the absence of $\alpha \mathrm{A}$-crystallin (Figure 1C).

These data suggested acceleration of the progressive neurodegeneration of the retina, specifically in the absence of $\alpha \mathrm{A}$ - but not $\alpha \mathrm{B}$-crystallin, and prompted us to assess how retinal function is affected. Consistent with previous reports (25), we observed that the amplitude of the dark-adapted (scotopic) b-wave of the electroretinogram (ERG) of WT animals, recorded at $+1.09 \log \mathrm{cd} \times \mathrm{s} / \mathrm{m}^{2}$ and representing the combined rodcone response, was progressively reduced by diabetes (Figure 1D). While this reduction was detectable in WT mice after 4 weeks, $\alpha$ A-crystallin-KO mice demonstrated a reduction in the scotopic $b$-wave amplitude as early as 2 weeks after diabetes induction (Figure 1D). While $\alpha$ B-crystallin loss did not affect cell survival and retinal thickness, it is associated with early decreased retinal function. This suggests that $\alpha \mathrm{B}$-crystallin, while not critical for retinal cell survival, is important for retinal function. The lack of effect of diabetes on the amplitude of the a-wave (data not shown) as well as on the light-adapted (photopic) responses (Figure $1 \mathrm{E}$ ) is consistent with previous reports (25) and supports a primary effect on the inner retina.

$\alpha A$-crystallin expression is regulated both at the transcriptional and posttranscriptional level. RNA and proteins were extracted from human retinal tissue of nondiabetic donors as well as diabetic donors with or without clinical signs of DR. Expression analysis was performed on central and peripheral retina samples, as diabetes affects those regions differently. $\alpha \mathrm{A}$-crystallin transcript levels were increased in the central retina of donors with diabetes and in the peripheral retina of diabetic donors with DR (Figure 2A). Western blot analysis showed that both $\alpha \mathrm{A}$ - and $\alpha \mathrm{B}$-crystallin protein levels were affected in retinal tissues from human donors with diabetes. $\alpha$-Crystallins levels were increased in the central retina of donors with diabetes and, even more so, in donors with DR (Figure 2B). A similar trend was observed in the peripheral retina, but it did not reach statistical significance.

$\alpha A$-crystallin is expressed in retinal neuron and glial cells. Our previous study in diabetic rats with a pan-specific antibody recognizing both $\alpha$-crystallins revealed primary expression in the inner retina (6); hence, we colabeled with specific markers of Müller glial cells (MGCs; glutamine synthetase) and ganglion cells (neurofilament-H). Immunostaining on retinal tissue from WT animals confirmed that $\alpha \mathrm{A}-$ and $\alpha \mathrm{B}$-crystallin were barely detectable in nondiabetic animals (Figure 2C). Similar analysis performed on diabetic tissues revealed increased $\alpha \mathrm{A}$-crystallin immunoreactivity. The signal partially colocalized with glutamine synthetase, indicating that $\alpha \mathrm{A}$-crystallin is highly expressed by MGCs, especially in their end feet (Figure $2 \mathrm{D}$, arrows). Costaining with neurofilament-H revealed that $\alpha \mathrm{A}$-crystallin was also expressed in ganglion cells (Figure 2D, arrowheads). The specificity of the signal was demonstrated by an absence of immunoreactivity when using retina and lenticular tissue from KO animals (Supplemental Figure 2). Colocalization of $\alpha$ A-crystallin and glial fibrillary acidic protein (GFAP), a marker of astrocytes and activated Müller cells, demonstrated a similar accumulation of $\alpha$ A-crystallin in activated Müller cells in diabetic human donors (Figure 2, E and F, arrows). Partial colocalization with neurofilament-H (Figure 2F, arrowheads) also confirmed expression by ganglion cells. The cellular localization of $\alpha \mathrm{B}$-crystallin showed a very similar pattern, with a slightly more diffuse signal partially colocalizing with GFAP (Supplemental Figure 3) and neurofilament-H (data not shown).

$\alpha A$-crystallin compensates for loss of B-crystallin expression. Analysis of the expression of $\alpha \mathrm{A}$-crystallin in $\alpha \mathrm{B}$-crystallin-KO mice and vice versa revealed a basal induction of $\alpha \mathrm{A}$-crystallin in the $\alpha \mathrm{B}$-crystallin-KO mice, animals that showed reduced retinal neurodegeneration (Figure 3, A and B). Conversely, $\alpha \mathrm{A}$-crystallin-KO mice, which showed enhanced cell death under diabetes conditions showed no induction of 
A ฮ

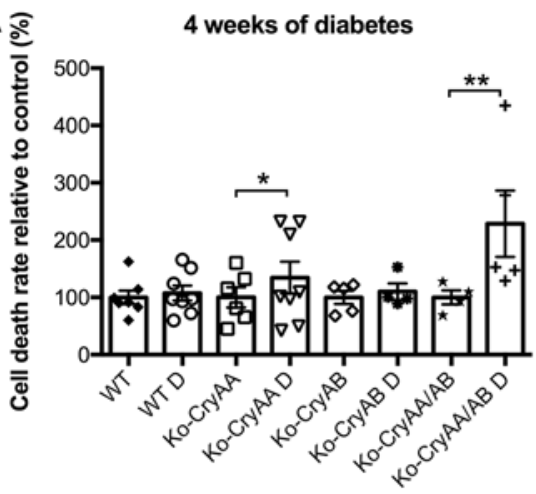

B

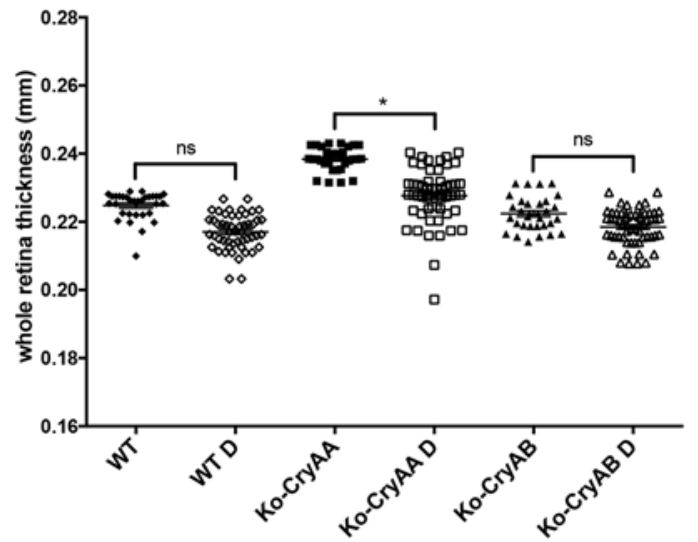

D

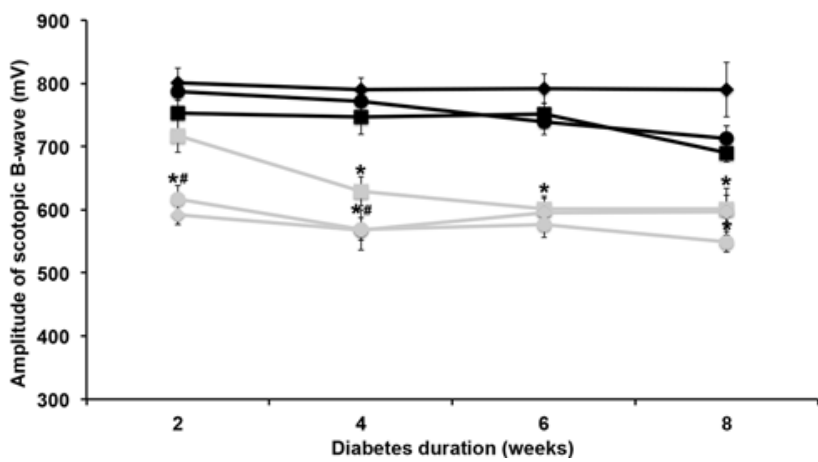

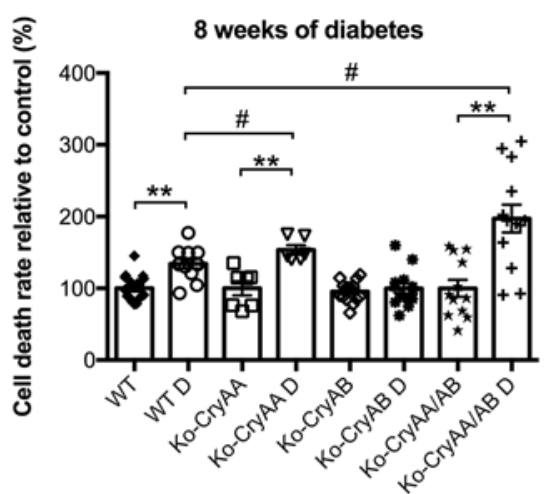

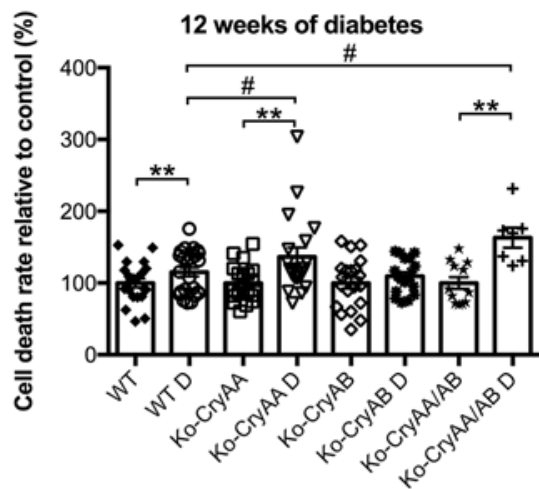

C

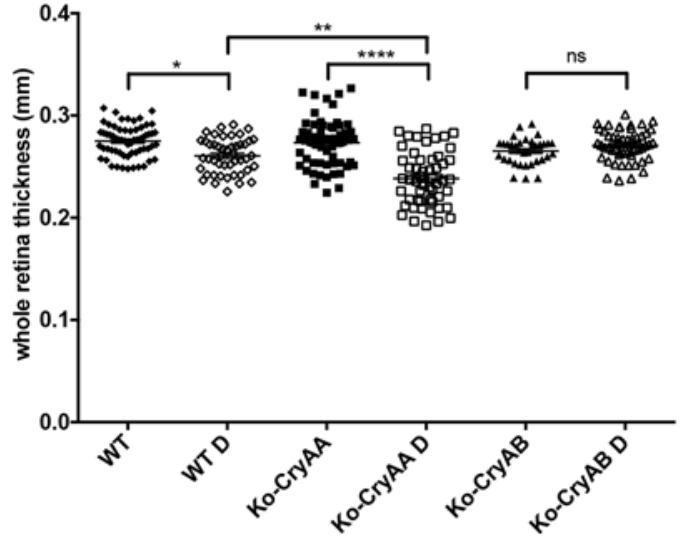

E

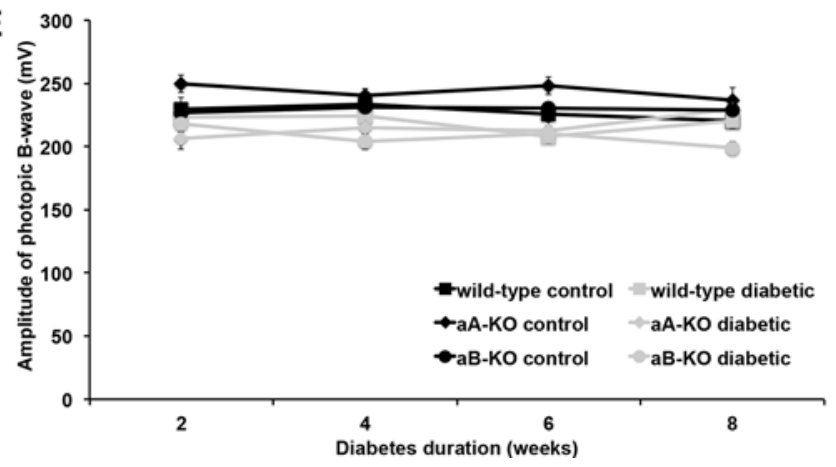

Figure 1. Retinal morphology and function of $\alpha$-crystallin-KO mice. Graphic representation of retinal cell death, measured by DNA fragmentation ELISA in whole retinas from WT, $\alpha \mathrm{A}$-crystallin-KO (Ko-CryAA), $\alpha \mathrm{B}$-crystallin-KO (Ko-CryAB), and double-KO (Ko-CryAA/AB) mice at 4, 8, and 12 weeks of diabetes (A). Retinal thickness was measured by optical coherence tomography (OCT) after 2 weeks (B) and by microscopy using histological sections after 4 weeks (C) of diabetes in the same experimental groups. Graphic representation of retinal function, assessed by electroretinogram (ERG) in the same animals, depicting the scotopic b-wave (D) and photopic b-wave amplitude (E) in diabetic WT (gray squares), $\alpha A$-crystallin-KO (gray diamonds), and $\alpha B$-crystallinKO (gray circles) mice compared with nondiabetic controls (black labels) 2, 4, 6, and 8 weeks after diabetes induction. The combined rod-cone responses recorded at $+1.09 \log \mathrm{cd} \times \mathrm{s} / \mathrm{m}^{2}$ are reported. Early cataract formation precludes the use of OCT and ERG in double-KO mice. ${ }^{*} P \leq 0.05,{ }^{*} P \leq 0.01$, significantly different from nondiabetic WT mice. ${ }^{\#} P \leq 0.05$, significantly different from WT diabetic mice. For each time point, a minimum of 8 mice was used for each group reported. Statistical analysis was performed by 1-way ANOVA followed by Student-Newman-Keuls test. D, diabetic.

$\alpha \mathrm{B}$-crystallin at baseline. Together, these data further support the protective role of $\alpha \mathrm{A}$-crystallin induction during the early stages of diabetes.

Lack of $\alpha$ A-crystallin enhances diabetes-induced ER stress in the retina. We assessed how lack of $\alpha \mathrm{A}$ - or $\alpha \mathrm{B}$-crystallin affected the UPR; we began by examining the central key regulator eukaryotic initiation factor $\alpha$ (eif2 $\alpha$ ) and its phosphorylation. Consistent with the protective role of $\alpha \mathrm{A}$-crystallin, both $\alpha \mathrm{A}$-crystallin-KO and double-KO mice showed increased eif2 $\alpha$ phosphorylation, characteristic of increased ER stress (Figure 3C). This alteration was further associated with increased expression of C/EBP homologous protein (CHOP), another downstream effector of the protein kinase RNA-like endoplasmic reticulum kinase (PERK) branch of ER stress, which is directly involved in ER stress-mediated apoptosis, (Figure 3D). 
A
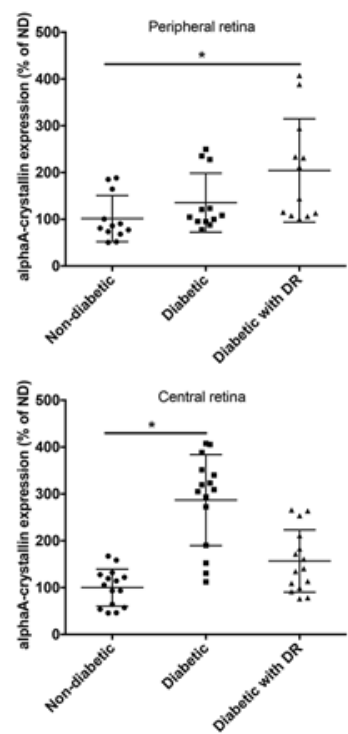
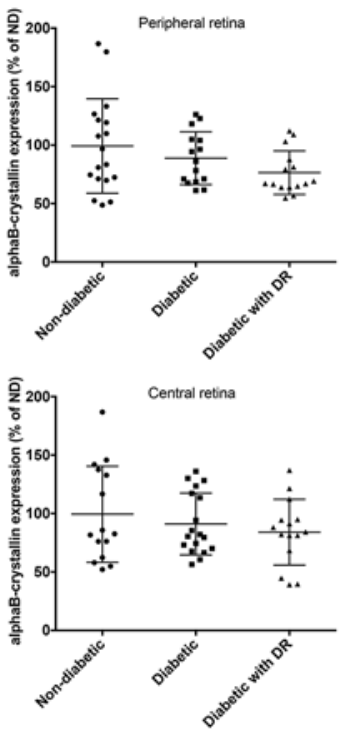

B
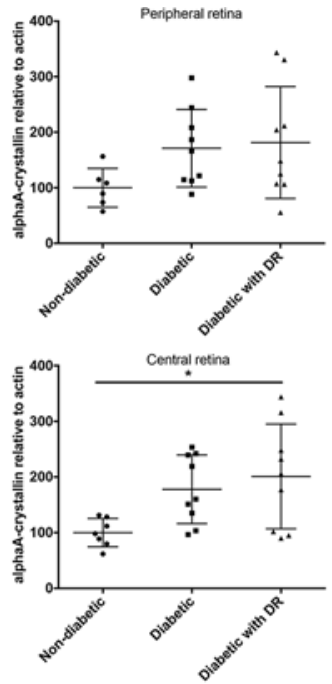

$\alpha$ A-crystallin ーレーーーー Actin $--1--$ $\frac{\text { ND D DR ND D DR }}{\text { Peripheral }} \frac{1}{\text { Central }}$
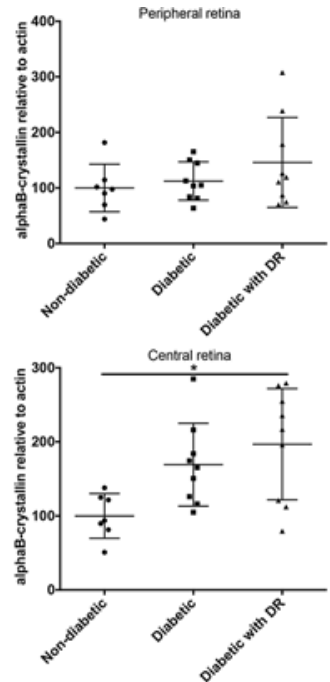

$\alpha$ B-crystallin -

Actin $-\longrightarrow-C$ $\frac{\text { ND D DR ND D DR }}{\text { Peripheral }} \frac{\text { Central }}{\text { Con }}$

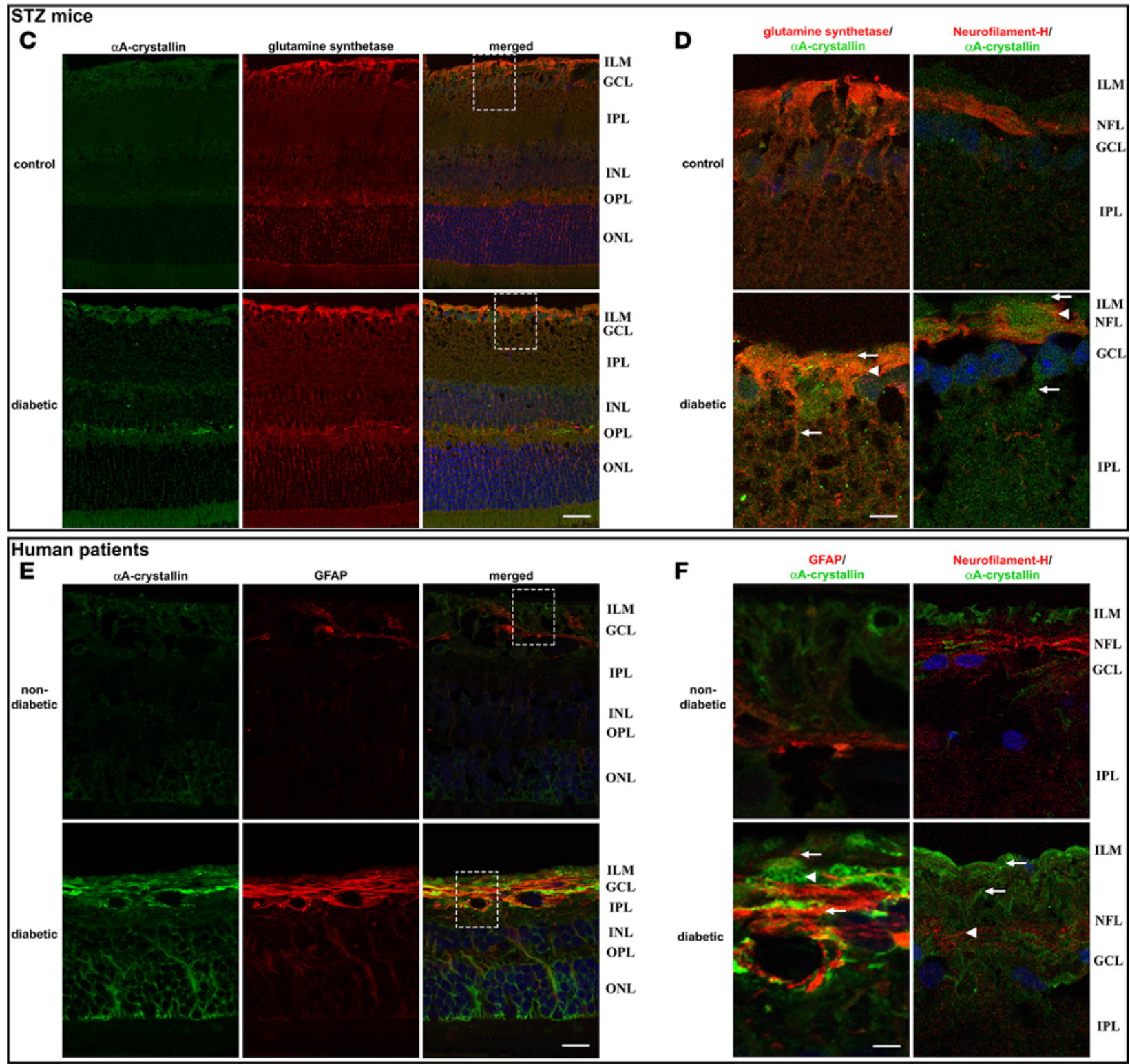


Figure 2. Cell-specific upregulation of $\alpha$-crystallins in diabetic patients with and without diabetic retinopathy and in diabetic rodents. The expression of $\alpha$-crystallins in the central and peripheral regions of the retina of human donors was analyzed by quantitative real-time PCR (A), immunoblot (B), and immunohistochemistry (C-F). Graphic representations and representative Western blot images of $\alpha$-crystallins levels in human donors, nondiabetic (ND; $n=7$ ) or diabetic without retinopathy $(D ; n=9)$ or with diabetic retinopathy (DR; $n=9$ ), are shown. Crystallin expression is presented normalized to actin levels and relative to the expression in nondiabetic donors ( ${ }^{*} P \leq 0.05$, significantly different from nondiabetic donors). Statistical analysis was performed by 1-way ANOVA followed by Student-Newman-Keuls test. Cellular localization of $\alpha$ A-crystallin (green) was assessed by immunofluorescent staining on retinal cross-sections from nondiabetic control and diabetic WT mice $(\mathbf{C}$ and $\mathbf{D} ; n=6)$ and nondiabetic and diabetic donors with diabetic retinopathy $(\mathbf{E}$ and $\mathbf{F} ; n=4)$. Coimmunostaining with specific markers of Müller glial cells (glutamine synthetase, $\mathbf{C}$ and $\mathbf{D}$, or glial fibrillary acidic protein [GFAP], $\mathbf{E}$ and $\mathbf{F}$, red) and ganglion cells (neurofilament-H, $\mathbf{D}$ and $\mathbf{F}$, red) reveals colocalization of $\alpha$ A-crystallins with Müller glial cells and partially with ganglion cells in diabetic animals (D) and human donors with DR (F). Nuclei were counterstained with Hoechst (blue). Higher-magnification images of relevant regions (marked by a dash square) show increased expression of $\alpha$ A-crystallins in Müller glial cells (arrows) and ganglion cells (arrowheads) during diabetes (scale bar: $20 \mu \mathrm{m}$ [C and E]; $4 \mu \mathrm{m}$ [D and F]). ILM, inner limiting membrane; GCL, ganglion cell layer; IPL, inner plexiform layer; INL, inner nuclear layer; OPL, outer plexiform layer; ONL, outer nuclear layer; NFL, nerve fiber layer.

Phosphorylation of $\alpha A$-crystallin on residue 148 is dramatically reduced by diabetes. $\alpha \mathrm{B}$-crystallin function is highly regulated by PTM, and our previous studies showed that both $\alpha \mathrm{A}$ - and $\alpha \mathrm{B}$-crystallins are targeted by PTMs in the context of diabetes (6). Using tandem mass spectrometry, we detected phosphorylation of serine residues $122,148,155$, and 173 of $\alpha \mathrm{A}$-crystallin in normal rodent retina. After isolating by gel separation after immunoprecipitation the two main $\alpha$ A-crystallin products detected by immunoblot analysis (see Figure $3 \mathrm{~A}$ ), we used multiple reaction monitoring to assess the effect of diabetes on this phosphorylation. This analysis showed that the relative amount of phosphorylation on Ser148 was reduced on average by $40 \%-50 \%$ after 3 months of diabetes (Figure 4 A). Similarly, when Thr148 phosphorylation was analyzed in human tissues, a reduction of more than $80 \%$ was observed in donors with DR for both major products of $\alpha \mathrm{A}$-crystallin detected by Western blot (Figure 4B). Interestingly, donors without retinopathy only showed a trend of a reduction of phosphorylation on that site.

Phosphorylation of $\alpha$ A-crystallin on residue 148 controls its neuroprotective function. To test the effect of this phosphosite on the neuroprotective function of $\alpha \mathrm{A}$-crystallin in retinal neurons, we turned to a cell culture model. Differentiated R28 rat retinal neuron cells were transfected with constructs expressing WT, the phosphomimetic (148D), or the nonphosphorylatable (148A) mutant of human $\alpha \mathrm{A}$-crystallin. Western blot and immunofluorescence analysis of the transfected cells showed that all 3 constructs lead to a similar transfection efficiency and level of expression of A-crystallin (Figure 4D, red channel). While the phosphomimetic $148 \mathrm{D}$ mutant was just as protective as the WT protein, the nonphosphorylatable 148A mutant showed an almost complete lack of protection, consistent with a key regulatory function of this phosphorylation (Figure 4, C and D). Further analysis demonstrated that this regulation of apoptosis was at least partially caspases-3/7 dependent (Figure $4 \mathrm{E}$ ).

$\alpha A$-crystallin regulation of ER stress in retinal neurons is dependent on its phosphorylation on residue 148 . Consistent with the in vivo data, a tempered ER stress response was observed in retinal neuronal cells overexpressing WT $\alpha$ A-crystallin in response to metabolic stress. Serum deprivation elicited an increase in ER stress in retinal neurons similar to the one seen after exposure with tunicamycin, a known ER stress inducer (Figure 5, A and B, EV control and 24h T lanes). This increase was significantly reduced in neurons overexpressing WT $\alpha$ A-crystallin, as demonstrated by reduced levels of phosphorylated eif $2 \alpha$ and nuclear activating transcription factor 4 (ATF4) levels, both downstream targets of the PERK-mediated branch of the UPR (Figure 5, A and B). Immunofluorescent analysis of the upstream regulator-binding immunoglobulin protein (BIP) and the chaperone protein disulfide isomerase (PDI; Figure 5, C and D) and splicing levels of X-box-binding protein-1 (XBP-1), the downstream effector of the inositol-requiring enzyme $\alpha$ (IRE1 $\alpha$ ) branch (Figure $5 \mathrm{E}$ ), also revealed a strong attenuation of ER stress by WT $\alpha \mathrm{A}$-crystallin overexpression. Similar attenuation of ER stress was observed with the phosphomimetic but not the nonphosphorylatable $\alpha \mathrm{A}$-crystallin mutant, confirming the central role of T148 phosphorylation.

Since $\alpha \mathrm{A}$-crystallin is highly expressed by MGCs, we evaluated the ER stress response in MIO-M1 (data not shown) and $\mathrm{rMC} 1$ cells overexpressing $\alpha \mathrm{A}$-crystallin $\mathrm{WT}$ or the distinct $\alpha \mathrm{A}$ variants. Unlike in retinal neurons, we found no significant effects of $\alpha A$-crystallin on ER stress, although an increase in ER stress upon treatment could be detected, albeit smaller than in retinal neurons (Supplemental Figure 4).

$\alpha A$-crystallin secreted by retinal glial cells protects neurons, an effect dependent on its phosphorylation on residue 148. MGCs are activated in diabetes, a phenomenon that leads to expression and release of trophic factors. Exogenous $\alpha \mathrm{A}$-crystallin can be protective, so we sought to determine if $\alpha \mathrm{A}$-crystallin itself can be secreted by MGCs and thus protects retinal neurons from metabolic stress. Immunoblotting revealed significant levels of 
A WT

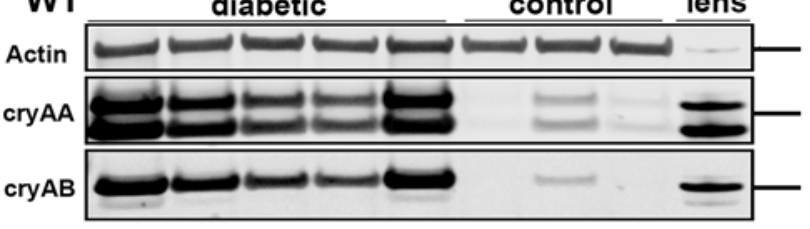

KO-cryAA diabetic control lens

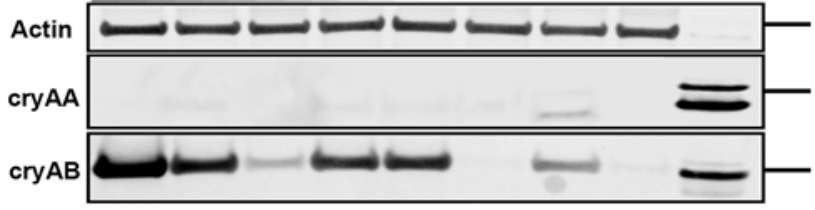

B

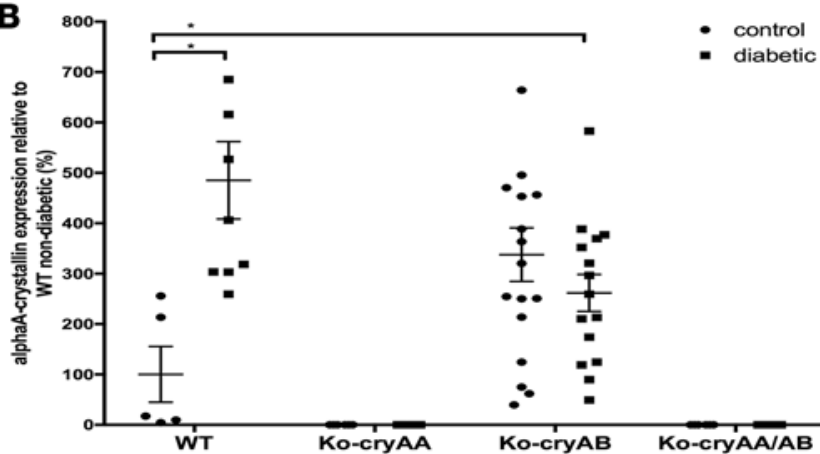

C

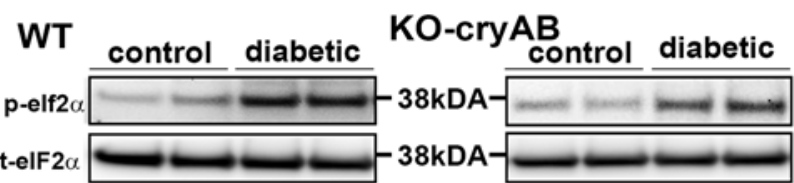

KO-cryAA control diabetic KO-cryAA/AB diabetic

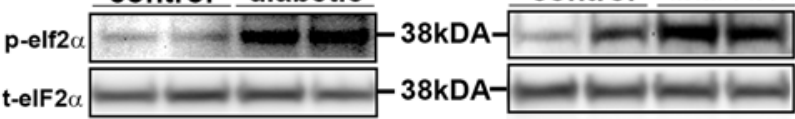

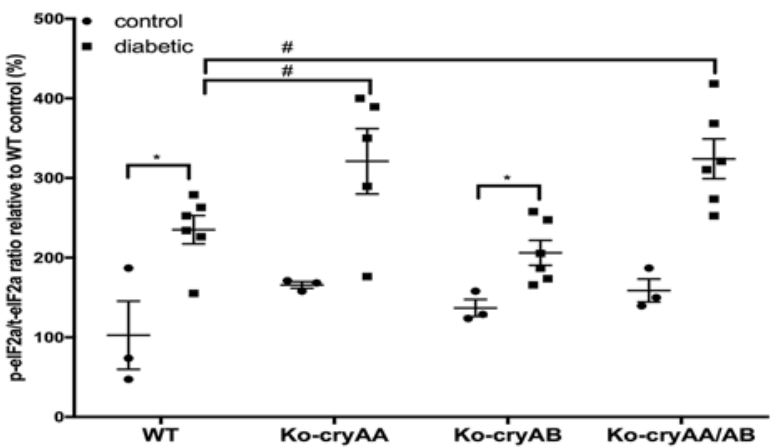

KO-cryAB diabetic control lens

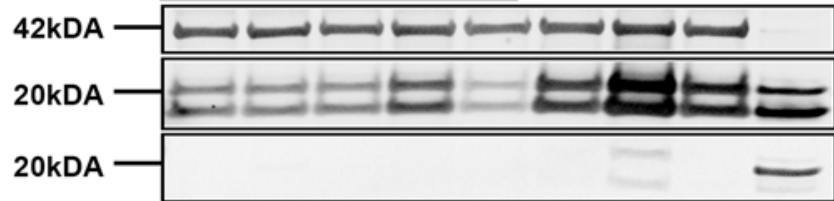

KO-cryAA/AB diabetic control lens
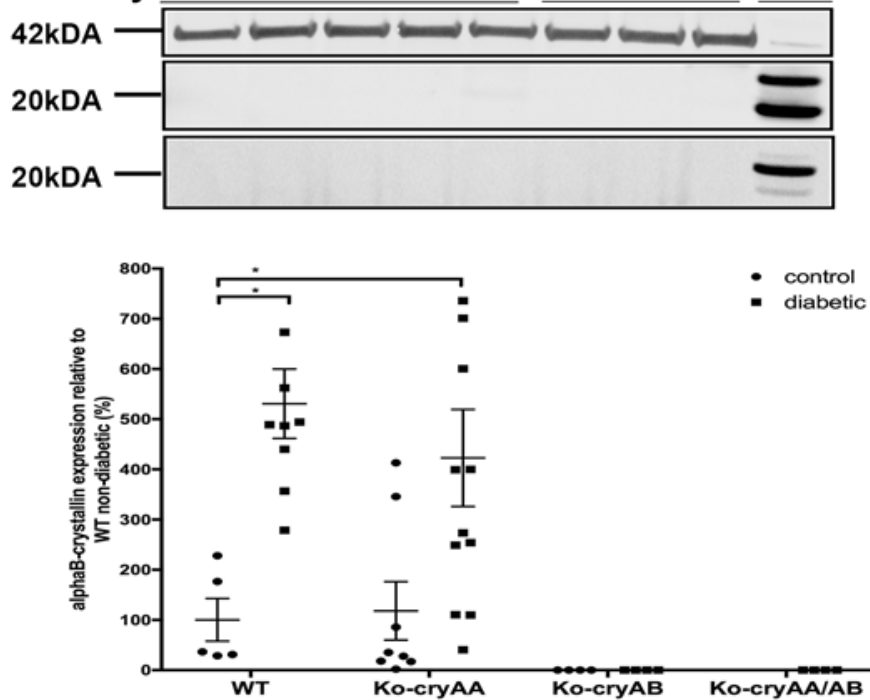

D
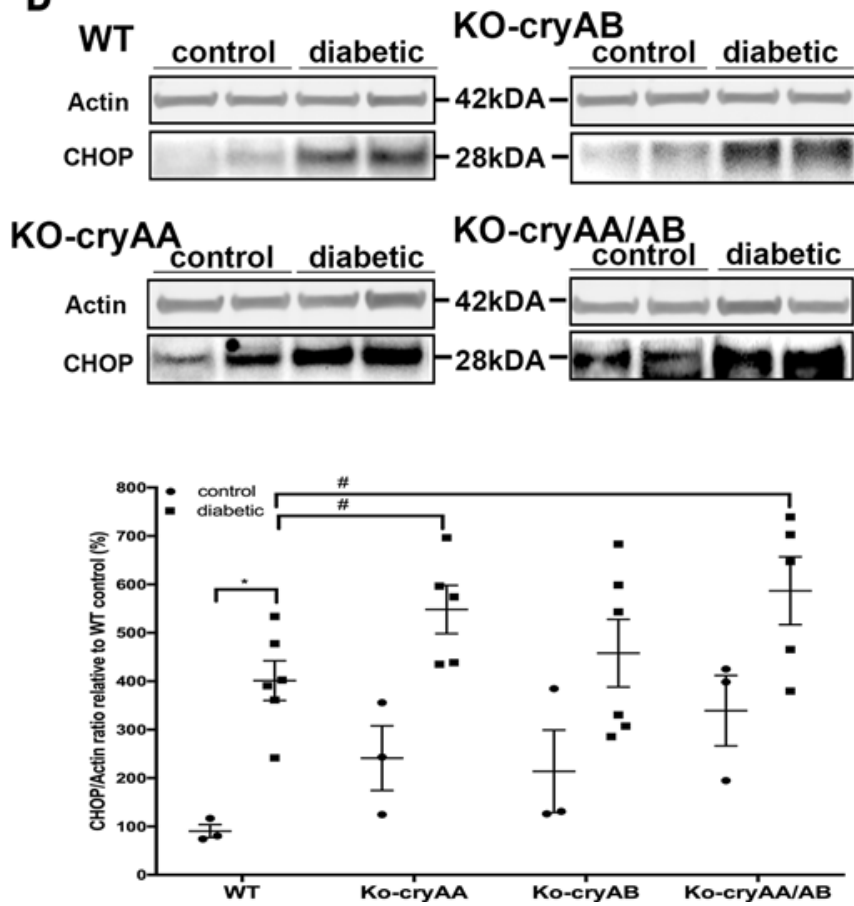

Figure 3. Reduced cell death in $\alpha \mathrm{B}$-crystallin-KO mice is associated with basal induction of $\alpha \mathrm{A}$-crystallin and alleviation of diabetes-induced endoplasmic reticulum stress by $\alpha$ A-crystallin. $\alpha$-Crystallin expression in the retina was assessed by immunoblot analysis after 12 weeks of diabetes. Representative images of immunoblots for $\alpha \mathrm{A}$ - and $\alpha \mathrm{B}$-crystallin (cryAA and cry $\mathrm{A} B$ ) $(\mathbf{A})$ and graphic representations of the corresponding quantification (B) are shown. Endoplasmic reticulum (ER) stress levels were assessed in retinal lysates by immunoblot analysis of eukaryotic initiation factor $\alpha$ (eif2 $\alpha$ ) phosphorylation as well as C/EBP homologous protein (CHOP) induction. Representative images of immunoblots for p-elF2 $\alpha$ as well as total protein (t-elF2 $\alpha)$ (C) and actin and CHOP (D) are shown. Retinal lysates from WT, $\alpha A$-crystallin-KO (Ko-cryAA), $\alpha B$-crystallin-KO (Ko-cryAB), and double-KO (Ko-cryAA/AB) diabetic mice and nondiabetic control littermates were compared ( $\mathbf{A}$ and $\mathbf{B}, n=10$ for diabetics, $n=6$ for controls; $\mathbf{C}$ and $\mathbf{D}, n=6$ for diabetics, $n=3$ for controls). ${ }^{*} P \leq 0.05$, significantly different from nondiabetic WT mice. ${ }^{*} P \leq 0.05$, significantly different from WT diabetic mice by 1-way ANOVA followed by Student-Newman-Keuls test. 

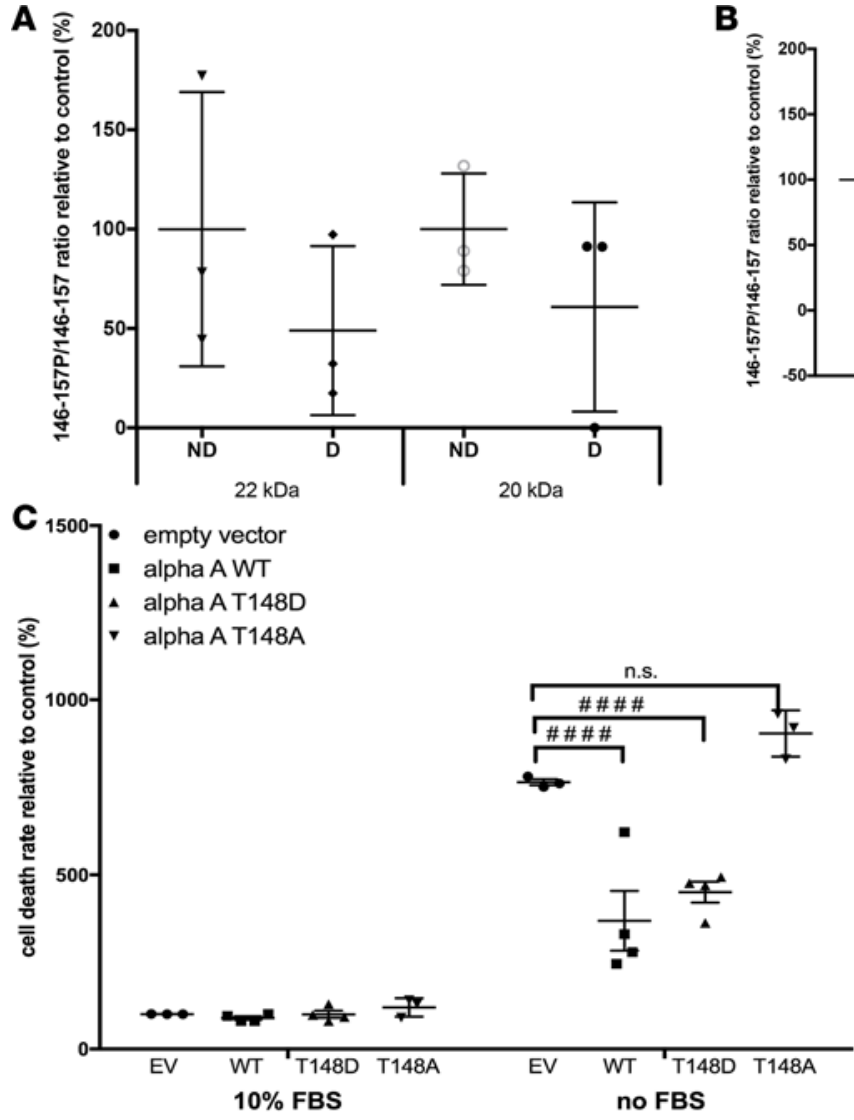

E

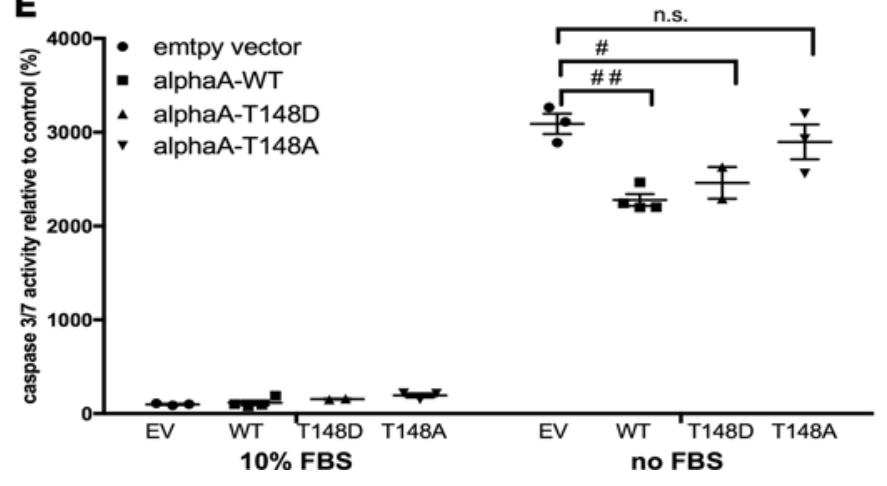

B
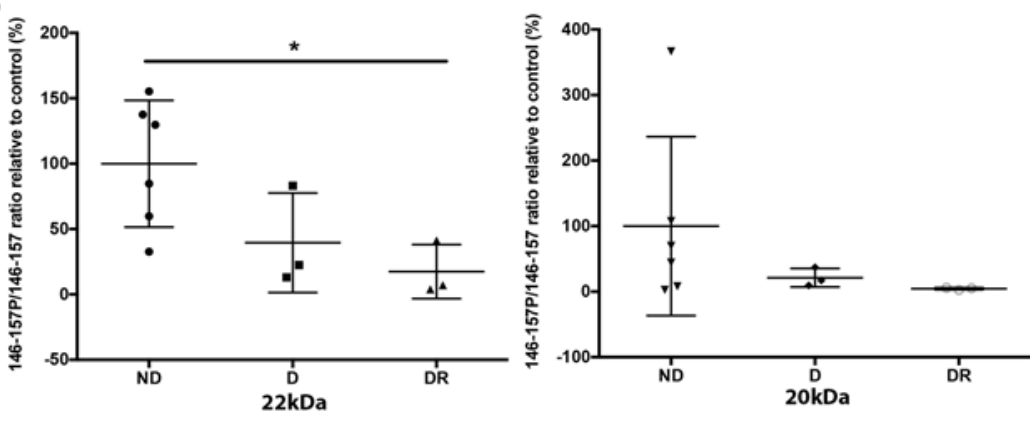

D
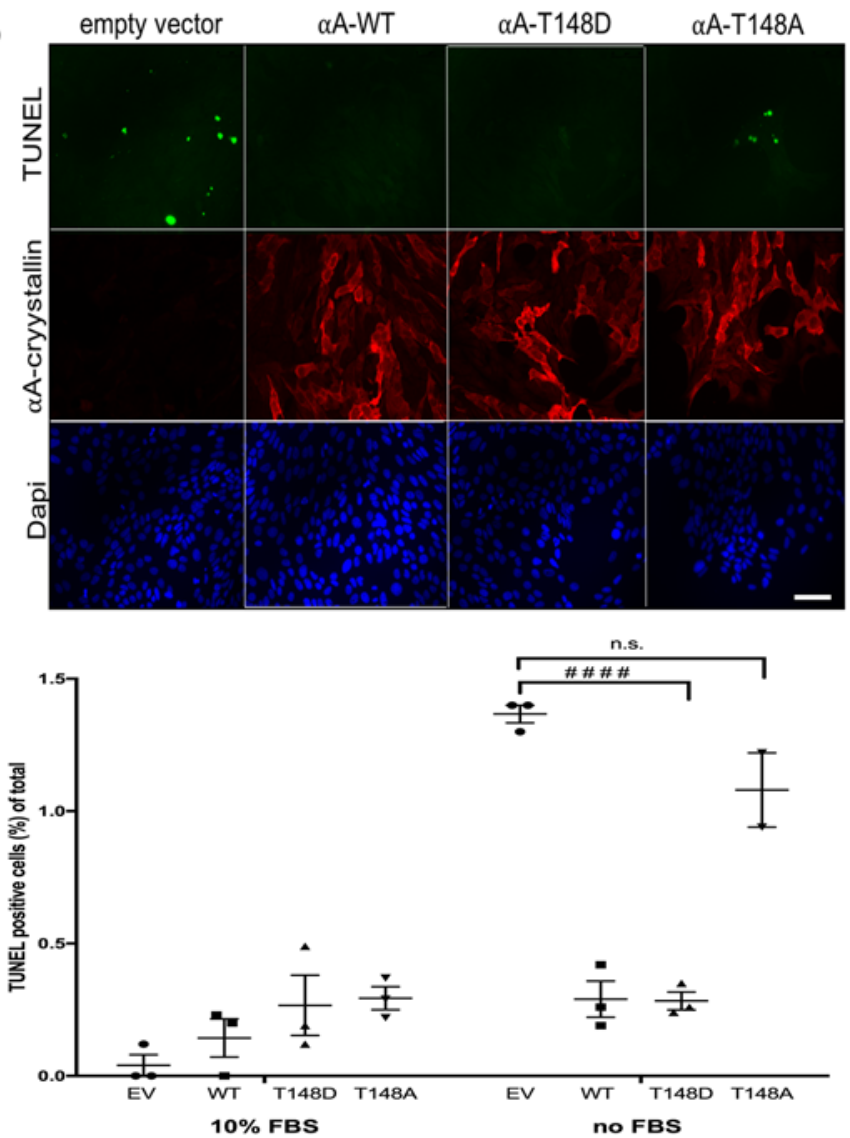

Figure 4. Phosphorylation on serine/threonine residue 148 of $\alpha$ A-crystallin, which is reduced by diabetes, regulates its neuroprotective function. The 2 main $\alpha \mathrm{A}$-crystallin products identified by immunoblot (20 and $22 \mathrm{kDa}$ ) were immunoprecipitated from mouse and human retinal lysates and analyzed by mass spectrometry (A and B). The level of phosphorylation of the 146-157 peptide was analyzed by multiple reaction monitoring, and the ratio of phosphorylated to nonphosphorylated peptide is graphically represented for (A) 12-week diabetic $(D, n=3)$ or nondiabetic mice (ND, $n=$ 3 ) and (B) nondiabetic (ND, $n=6$ ), diabetic without retinopathy $(D, n=3)$, or diabetic with retinopathy human donors $(\mathrm{DR}, n=3)$. ${ }^{*} P \leq 0.05$, significantly different from nondiabetics. (C and E) Effect of $\alpha$ A-crystallin phosphorylation on the threonine residue 148 on retinal neuronal cell survival. Serum deprivation (no FBS) induced cell death was measured in neurons transfected with the empty vector (EV) versus neurons overexpressing WT $(\alpha A-W T)$, the phosphomimetic mutant $(\alpha A-T 148 D)$, or the nonphosphorylatable mutant $(\alpha A-T 148 A)$ of $\alpha A$-crystallin on threonine 148. Cell death was assessed by DNA fragmentation ELISA (C), TUNEL staining (D), and caspase $3 / 7$ activity assay (E). Representative immunofluorescence images of serum-deprived transfected neurons are shown for the TUNEL data (TUNEL in green, $\alpha A$-crystallin in red, and nuclei in blue; scale bar: $50 \mu \mathrm{m}$ ), while

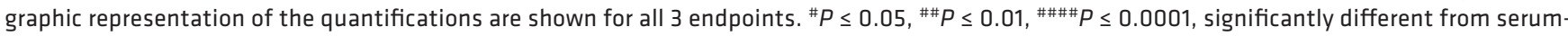
starved EV-transfected cells. Each endpoint was measured on a minimum of 3 technical replicates in 3 independent experiments (2 for TUNEL). Statistical analysis was performed by 1-way ANOVA followed by Student-Newman-Keuls test. 
A

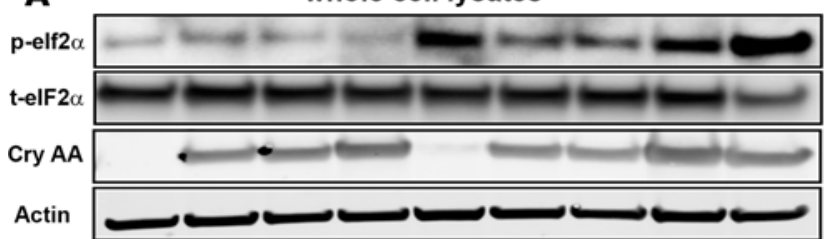

EV WT T148D T148A EV WT T148D T148A 24h T untreated

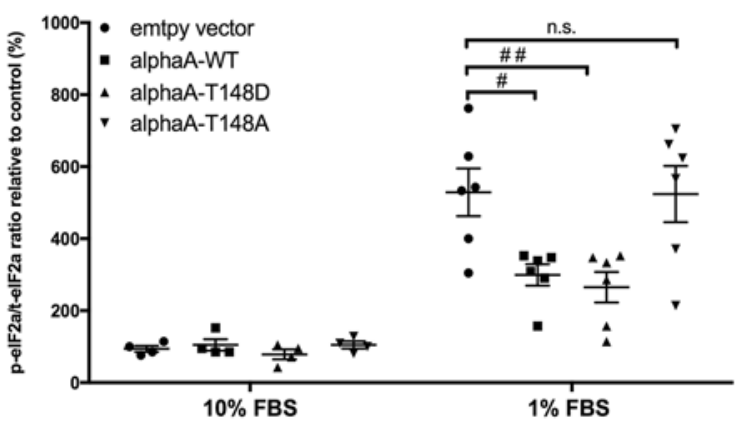

C

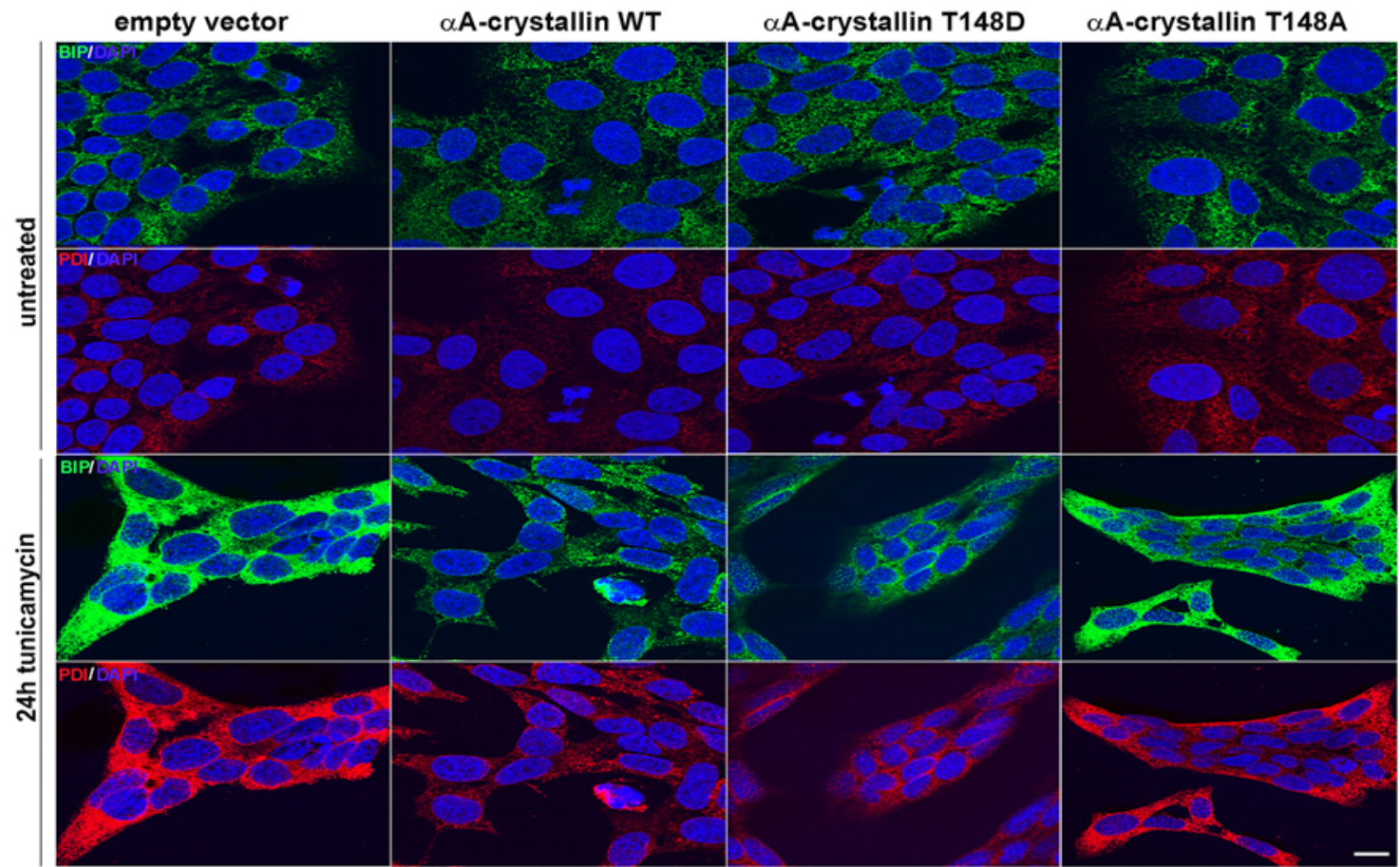

D

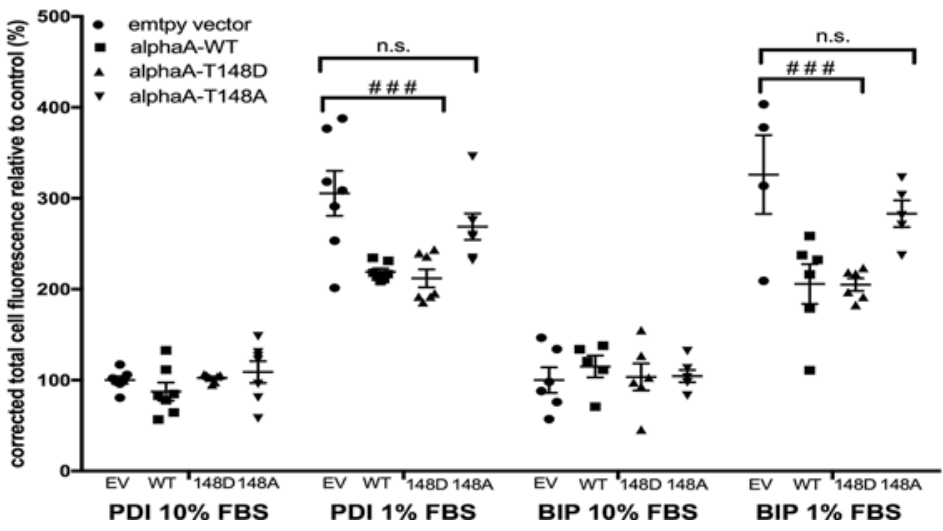

B

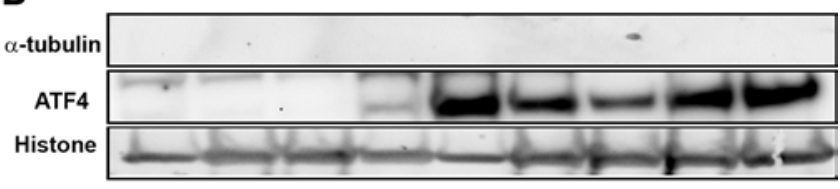

EV WT T148D T148A EV WT T148D T148A 24h T untreated

$1 \%$ FBS

+ ctrl
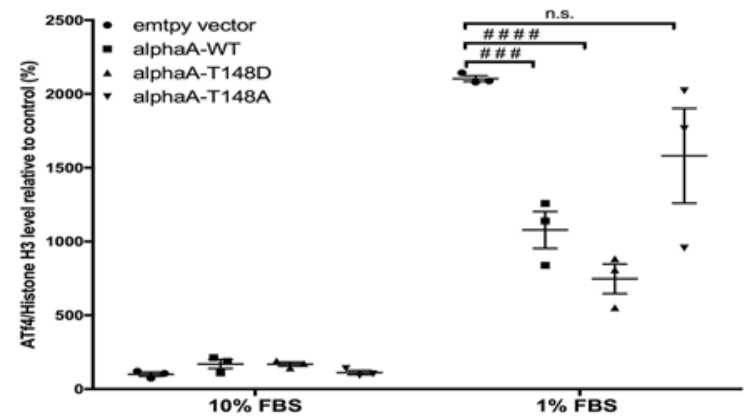

$1 \%$ FBS

E

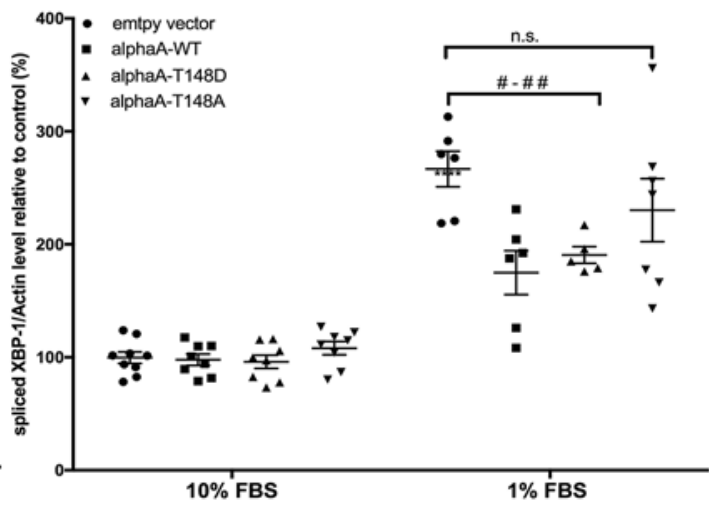


Figure 5. $\alpha$ A-crystallin attenuates endoplasmic reticulum stress in retinal neurons, an effect regulated by its phosphorylation on the 148 residue. Retinal neurons were transfected with vectors overexpressing WT ( $\alpha \mathrm{A}-\mathrm{WT}$ ), the phosphomimetic mutant ( $\alpha \mathrm{A}-\mathrm{T} 148 \mathrm{D})$, the nonphosphorylatable mutant $(\alpha A-T 148 A)$ of $\alpha A$-crystallin on threonine 148 , or the empty vector control (EV). The cells were then subjected to serum starvation (1\% FBS) or tunicamycin $(0.5 \mu \mathrm{g} / \mathrm{ml}$, positive control), and the endoplasmic reticulum (ER) stress response was assessed by analysis of eukaryotic initiation factor $\alpha$ (eif $2 \alpha)$ phosphorylation (A), activating transcription factor 4 (ATF4) nuclear translocation (B), binding immunoglobulin protein (BIP) and protein disulfide isomerase $(\mathrm{PDI})$ induction (C and $\mathbf{D})$, and X-box binding protein-1 (XBP-1) splicing (E). Representative images of the immunoblots and the corresponding graphic representation of the quantifications are shown for p-elF $2 \alpha$, total-elf $2 \alpha$, actin, and $\alpha$ A-crystallin (cryAA) (A) and ATF4, Histon H3, and $\alpha$-tubulin (B). Representative images of the immunofluorescent staining obtained for BIP (green), PDI (red), and Hoechst (blue) (C) and the corresponding graphic representation of the relative quantification (D) (scale bar: $10 \mu \mathrm{m}$ ). Graphic representation of the quantitative real time PCR for spliced XBP-1 (E). Gene expression was normalized to the actin-encoding gene Actb. ${ }^{P} P \leq 0.05,{ }^{\#} P \leq 0.01$, \#\#\# $P 0.001$, \#\#\# $P \leq 0.0001$, significantly different from serum deprived EV-transfected cells. Each endpoint was measured on a minimum of 3 technical replicates in 3 independent experiments. Statistical analysis was performed by 1 -way ANOVA followed by Student-Newman-Keuls test.

$\alpha \mathrm{A}-$ crystallins in the cell lysate but also the culture media of MGCs overexpressing WT $\alpha \mathrm{A}$-crystallin (Figure $6 \mathrm{~A}$ ). We also determined that $\alpha \mathrm{A}$-crystallins are secreted at least in part within vesicular structures (e.g., exosomes), as approximately $50 \%$ of $\alpha \mathrm{A}$-crystallin was protected from digestion by proteinase $\mathrm{K}$ in absence of a permeabilizing agent (Triton $\mathrm{X}$ ), whereas the cytoskeleton protein actin was completely digested (Figure $6 \mathrm{~A})$. Consistent with a paracrine protective role of $\alpha \mathrm{A}-c r y s t a l l i n$, we showed that media from cells overexpressing either WT or the phosphomimetic form of $\alpha \mathrm{A}$-crystallin, but not the nonphosphorylatable mutant of $\alpha \mathrm{A}$-crystallin, was able to partially rescue stress-induced neuronal cell death (Figure 6B). Consistent with a direct effect of secreted $\alpha \mathrm{A}$-crystallin, this protective effect was blocked by preincubation of the media with an antibody against $\alpha \mathrm{A}-$ crystallin (Figure $6 \mathrm{C}$ ).

\section{Discussion}

This study reveals the differential roles of $\alpha \mathrm{A}$ - and $\alpha \mathrm{B}$-crystallin proteins, demonstrates the critical role of $\alpha \mathrm{A}$-crystallin in neuroprotection of retinal cells during the early stages of diabetes, and characterizes a mechanism of regulation of this intrinsic protective pathway. Our work demonstrates for the first time to our knowledge how the protective function of $\alpha \mathrm{A}$-crystallin is strongly dependent on its phosphorylation pattern, which is dramatically altered in diabetic patients with DR. This work also reveals a paracrine mechanism of neuroprotection by $\alpha$ A-crystallin via its secretion by MGCs to promote survival of associated retinal neurons. Taken together our findings identify $\alpha \mathrm{A}$-crystallin and its phosphorylation as a candidate to target for the treatment of neurodegenerative diseases.

Our study demonstrates that $\alpha \mathrm{A}$-crystallin is predominantly expressed in ganglion and MGCs in diabetic patients. This pattern of expression is consistent with previous reports in animal models of diabetes $(6,26)$ as well as other neurodegenerative models primarily affecting the inner retina, such as ischemic neuropathies, optic nerve crush, and glaucoma (27). This expression pattern is consistent with studies suggesting a central role for $\alpha \mathrm{A}$ - and $\alpha \mathrm{B}$-crystallin in the neuroglial unit in neurodegenerative diseases. These studies showed that $\alpha$-crystallins played a key role in stroke and experimental autoimmune uveitis, in part through modulation of neuroinflammation by way of their role in glial cells $(12,14)$. At the crosstalk of neuroprotection and neuroinflammation regulation, another study showed that exogenous $\alpha \mathrm{B}$-crystallin could decrease microglial activation while also enhancing glial cell survival in a model of ischemic neuropathy (28). This key role of $\alpha$-crystallins has been suggested in glial cells throughout the central nervous system, including the spinal cord (29).

While these studies point out a very important potential for $\alpha$-crystallins in the treatment of neurodegenerative diseases, the regulation of the protective role of these proteins remained unclear. Indeed, it is important to understand why neurodegeneration still occurs in numerous diseases in which these proteins are upregulated. PTMs and their consequences on the chaperone function of $\alpha$-crystallins have been only minimally studied outside of the lens. We and others previously demonstrated a correlation between levels of expression and phosphorylation on serine residues 19, 45, and 59 of $\alpha \mathrm{B}$-crystallin in the retina in response to diabetes $(21,26)$. Serine residue 148 in rodents and threonine 148 in humans have been previously reported as phosphorylation sites of lenticular $\alpha$ A-crystallin (30) as well as in brain astrocytes in culture (31). The current study is, however, the first to our knowledge to report a physiological role and pattern of regulation of this phosphorylation in vivo under normal or pathological conditions. Indeed, we show here that phosphorylation on this residue is high in the retina under normal conditions, while it is dramatically reduced in diabetic donors, especially those with retinopathy. Using 
A
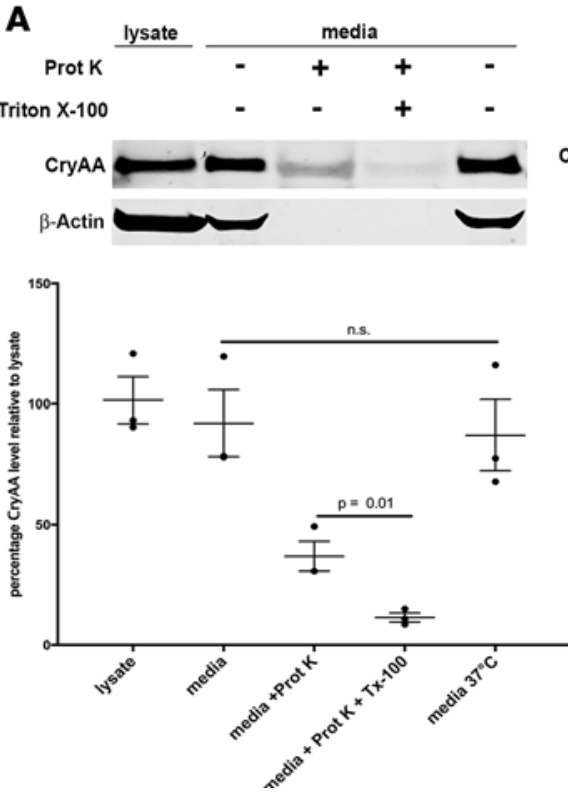

B
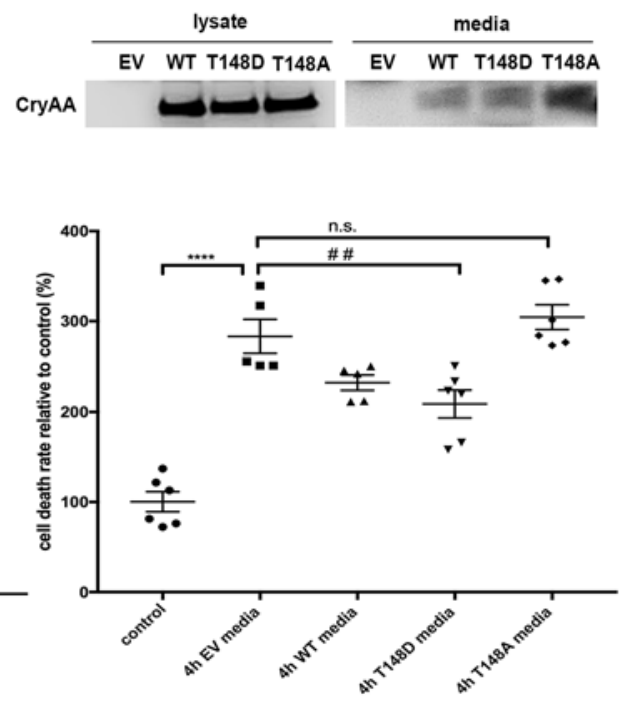

C
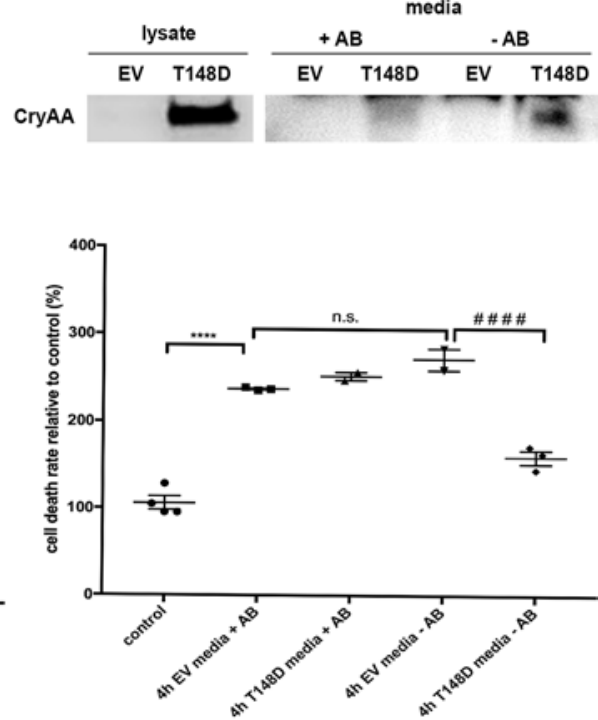

Figure 6. $\alpha$ A-crystallin, secreted by Müller glial cells, protects retinal neurons against serum deprivation-induced cell death. (A) Representative Western blot images of whole-cell lysate and the media of Müller glial cells transfected with a vector overexpressing $\alpha$ A-crystallin and corresponding graphic representation. The serum-free culture media were recovered after 24 hours, concentrated, and treated or not with proteases (proteinase $\mathrm{K}$ ) and permeabilizing agent Triton X-100 before the Western blot. (B and $\mathbf{C}$ ) Protective effect of secreted $\alpha \mathrm{A}$-crystallins on retinal neuronal survival. Retinal neurons were treated for 4 hours with serum-free concentrated media, recovered from Müller cells, transfected with the empty vector (EV) or $\alpha A-c r y s t a l l i n$ WT ( $\alpha A-W T$ ), the phosphomimetic mutant ( $\alpha A-T 148 D$ ), or the nonphosphorylatable mutant ( $\alpha A-T 148 A$ ) of $\alpha A$-crystallin on threonine 148 (B) or transfected with the EV and $\alpha A-T 148 D$ (C). (B) Representative Western blot images for $\alpha A$-crystallin on the neuronal cell lysates, processed for the DNA fragmentation ELISA after 4-hour incubation with the different crystallin-enriched media (lysate) and Western blot of the concentrated media from transfected Müller cells (media). Relative amounts are normalized by volume. The graph depicts the DNA fragmentation ELISA data. (C) Representative Western blot images for $\alpha A$-crystallin on the neuronal cell lysates, processed for the DNA fragmentation ELISA after 4 hours of incubation with $\alpha$ A-T148D crystallin-enriched media (lysate) with (+Ab) or without (-Ab) addition of an $\alpha A$-crystallin-neutralizing antibody before the 4-hour incubation or neurons incubated with media from EV-transfected Müller cells and Western blot of the corresponding concentrated media from transfected Müller cells (media). The graph depicts the DNA fragmentation ELISA data (bottom panel). Each endpoint was measured on a minimum of 3 technical replicates in at least 2 independent experiments.

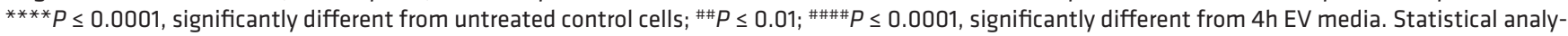
sis was by unpaired 2-tailed $t$ test (A) and 1-way ANOVA followed by Student-Newman-Keuls test (B and $\mathbf{C}$ ).

point mutants, we sought to compare the protective nature of phosphomimetic and nonphosphorylatable forms of $\alpha \mathrm{A}$-crystallin in retinal neurons in culture. While the phosphomimetic form of $\alpha \mathrm{A}$-crystallin showed a very strong protective effect, the nonphosphorylatable mutant was widely ineffective, clearly demonstrating a strong central regulatory role of the serine/threonine 148 phosphorylation and consistent with its dramatic decrease observed in diabetic donors with DR.

$\alpha$-Crystallins are widely known as chaperone proteins involved in the UPR, whether it is in the lens, the cardiovascular system, or the central nervous system $(32,33) . \alpha$-Crystallins are induced by cellular stress in numerous cell types and conditions to prevent accumulation of damaged or unfolded proteins, lower ER protein load, and enhance protein folding activity. These mechanisms are in part mediated by the UPR/ ER stress response. The current study demonstrated that the phosphorylation of A-crystallin on the serine/threonine 148 residue regulates the ER stress response associated with metabolic stress in retinal neurons. Indeed, overexpression of the phosphomimetic, but not the nonphosphorylatable, form of A-crystallin reduced neuronal apoptosis and ER stress concomitantly. Conversely, overexpression of $\alpha \mathrm{A}$-crystallin in retinal glial cells had no effect on ER stress, suggesting a different role of $\alpha \mathrm{A}$-crystallin in this cell type. The primary supportive role of retinal glial cells coupled with the previous demonstration of $\alpha \mathrm{B}$-crystallin secretion $(34,35)$ prompted us to assess a possible paracrine role of $\alpha \mathrm{A}$-crystallin expressed by glial cells. The current study demonstrates for the first time to our knowledge that glial cells can secrete $\alpha \mathrm{A}-\mathrm{crystallin}$ and that this secretion is associated with protection of neuronal cells. This finding is in line with a previous study showing that systemic administration of $\alpha \mathrm{B}$-crystallin greatly diminished disease progression in a model of multiple sclerosis (36). Altogether this is important, as it further supports the use of $\alpha$-crystallins for the treatment of neurodegenerative conditions, by either local or systemic administration. This secreted and paracrine 
protective role of $\alpha \mathrm{A}$-crystallin could help reveal its role in other glial cells, such as brain oligodendrocytes and astrocytes, in which its expression has been reported but without a clear understanding of its function.

In conclusion, this study unveils mechanisms that could be targeted for the treatment of DR and other neurodegenerative diseases as a means to promote neuronal survival.

\section{Methods}

Human donors. Ocular tissues were recovered from human cadavers within 9 hours of death, and retinas were immediately frozen until processing. Fragments of central and peripheral retina were isolated and processed for either RNA isolation (qPCR analysis) or protein analysis (Western blot and multiple reaction monitoring analysis) (see below for details). Tissues were obtained from 8 nondiabetic donors, 9 diabetics without retinopathy, and 9 diabetic donors with nonproliferative retinopathy, subgroups of which were used for the different procedures listed below. Retinopathy grading was confirmed by fundus photograph and OCT. None of the donors had proliferative retinopathy or diabetic macular edema. The groups were normalized for race $(87 \%$ [nondiabetic donors], 78\% [diabetics without retinopathy], and 67\% [diabetic donors with nonproliferative retinopathy] of European descent), sex (62\% [nondiabetic donors], 78\% [diabetics without retinopathy], and 50\% [diabetic donors with nonproliferative retinopathy] female), and age (mean of 66 [nondiabetic donors], 69 [diabetics without retinopathy], and 69 [diabetic donors with nonproliferative retinopathy] years old). While slightly greater, diabetes duration (10.2 for diabetics without retinopathy and 13.6 for diabetic donors with nonproliferative retinopathy) and HbA1c levels (6.46 for diabetics without retinopathy and 6.86 for diabetic donors with nonproliferative retinopathy) were not statistically different between the diabetic donors with and without retinopathy.

Induction of diabetes. Mice were housed under a 12-hour-light/dark cycle with free access to standard mouse chow and water. $\alpha \mathrm{A}$ - and $\alpha \mathrm{B}$-crystallin-KO mice were provided by $\mathrm{E}$. Wawrousek from the National Eye Institute (NEI, Bethesda, Maryland, USA) and were previously characterized and described (22, 23). Diabetes was induced by a total of 3 intraperitoneal injections of streptozotocin ( $50 \mathrm{mg} / \mathrm{kg}$; MilliporeSigma) dissolved in $10 \mathrm{mM}$ sodium citrate buffer, $\mathrm{pH} 4.5$, while age-matched nondiabetic controls were generated by injection of equivalent volumes of buffer alone. Mice were considered diabetic when exhibiting blood glucose levels $>13.9 \mathrm{mmol} / 1$ ( $250 \mathrm{mg} / \mathrm{dl}$; One-Touch meter; Lifescan). The various durations of diabetes were chosen because of the previously reported retinal alterations in diabetic rats (3, 37-40) and mice (41).

Retinal anatomy and visual function analysis. Mice were anesthetized with an intraperitoneal injection of ketamine $(93 \mathrm{mg} / \mathrm{kg})$ and xylazine $(8 \mathrm{mg} / \mathrm{kg})$. Pupils were dilated with topical phenylephrine $(2.5 \%)$ and tropicamide (1.0\%) followed by application of GenTeal Lubricant Eye Gel, $10 \mathrm{~g}$ (Alcon), or 2\% methylcellulose to keep the cornea moist and prevent opacification.

$O C T$. The SD-OCT images were obtained using the Bioptigen Spectral-Domain Ophthalmic Imaging System (SD OIS; Bioptigen Envisu R2200; Bioptigen Inc.). Imaging included averaged single B-scan and volume intensity scans, with images centered on the optic nerve head. Postimaging analysis was performed by manual assessment of all retinal layers using InVivoVueTM DIVER 2.4 software in a masked fashion (Bioptigen Inc.). The data are represented as an average value of the whole retina in the scattered plots provided.

Electroretinography. To assess rod- and cone-mediated retinal function, full-field ERGs were performed using the Espion e2 recording system (Diagnosys) as described previously (42). After overnight dark adaptation, and anesthesia as described above, pupils were dilated and corneal ERGs were recorded from both eyes using gold wire loops mounted in a contact lens electrode (Mayo Corporation). The dark-adapted ERGs were recorded at $-2.31 \log \mathrm{cd} \times \mathrm{s} / \mathrm{m}^{2}$ to record the rod isolated response and +1.09 $\log \mathrm{cd} \times \mathrm{s} / \mathrm{m}^{2}$ to record the combined rod-cone response. Ten to twenty-five responses were recorded at 3-60 seconds, depending upon the stimulus intensity. After 10 minutes of light adaptation to a white 32 $\mathrm{cd} / \mathrm{m}^{2}$ rod-suppressing background, light-adapted ERGs were recorded at $1.09 \log \mathrm{cd} \times \mathrm{s} / \mathrm{m}^{2}$.

Cell culture and transfection. Rat retinal neuronal cells (R28) were purchased from Applied Biological Material Inc. The R28 cell-line is derived from postnatal day 6 Sprague-Dawley rat retinal tissue immortalized with the 12S E1A gene of adenovirus and shown to express neurotransmitter receptors to dopamine, serotonin, glycine, acetylcholine, and glutamate (43). Cells were grown in DMEM containing $5 \mathrm{mM}$ glucose supplemented with $10 \%$ FBS (Flow Laboratories) and differentiated to neurons on laminin-coated plates with addition of cell-permeable cAMP (MilliporeSigma) as described previously (44).

Rat retinal Müller cells (rMC-1) were purchased from Applied Biological Material Inc. The rMC-1 cell line is derived from stable transformation of SV40 antigen into primary rat retinal Müller cells shown to 
express GFAP and cellular retinaldehyde-binding protein (CRALBP), two markers for Müller cells in the adult retina (45). Cells were maintained in DMEM containing $25 \mathrm{mM}$ glucose supplemented with $10 \% \mathrm{FBS}$.

Cells were transfected using the NeonÔTransfection System (Invitrogen) following the manufacturer's instructions. Briefly, cells were trypsinized and washed in PBS before being resuspended in 1001 resuspension buffer and electroporated. Cells were then plated in 96-well plates for DNA fragmentation and caspase assay or on coverslips for TUNEL staining. Thirty-six hours after transfection cells were placed in serumfree DMEM four hours before analysis. To determine the expression of ER stress markers, R28 and rMC-1 cells were treated with serum starvation ( $1 \% \mathrm{FBS})$ for 4 hours or tunicamycin $(0.5 \mu \mathrm{g} / \mathrm{ml})$ for 24 hours. Cells were then harvested for biochemical or immunocytochemical assays.

Cell death assays. Apoptosis was measured by Cell DNA Fragmentation ELISA (Roche Diagnostics) according to the manufacturer's instructions and as previously described (17). Briefly, after homogenizing the retinal tissue or cells in lysis buffer, samples were incubated and spun. $20 \mu 1$ of the supernatant, as well as of the positive and negative controls, were then transferred into the ELISA plate along with the immunoreagent complex. Following incubation and washes, the colorimetric solution was added and incubated until the colorimetric reaction developed. After adding the stop solution, the colorimetric signal was measured with a fluorescence plate reader (SpectraMax Gemini EM; Molecular Devices) with excitation at 405 and $490 \mathrm{~nm}$.

Cell death was also measured by TUNEL with horseradish peroxidase detection fixed cells as described previously (3). TUNEL-positive cell counts were calculated as percentages of total cell number at $\times 10 \mathrm{mag}$ nification using a light microscope (Leica).

Caspase-3/7 activity was measured using the ApoONE Assay (Promega) as described previously (44).

Subcellular fractionation. For immunoblot analysis of ATF4, transfected and treated rMC1 and R28 cells were homogenized in a buffer containing $10 \mathrm{mM}$ HEPES (pH 7.9), $2.5 \mathrm{mM} \mathrm{KCL}, 0.1 \mathrm{mM}$ EDTA (MilliporeSigma), $2 \mathrm{mM} \mathrm{MgCl}$, $1 \mathrm{mM}$ DTT (Calbiochem), 0.2\% NP-40, (Thermofisher) and protease inhibitor cocktail. After centrifugation at 3,500 $\mathrm{g}$ for 5 minutes, the supernatant containing the cytoplasmic protein was removed. The pellet containing nuclear protein was washed twice with ice-cold wash buffer $(10 \mathrm{mM}$ HEPES [pH 7.9], $20 \mathrm{nM} \mathrm{KCL,} 2 \mathrm{mM} \mathrm{MgCl}$, $0.1 \mathrm{mM}$ EDTA, $1 \mathrm{mM}$ DTT, and protease inhibitor cocktail) and resuspended in a buffer containing $20 \mathrm{mM}$ HEPES ( $\mathrm{pH} 7.9$ ), $630 \mathrm{mM} \mathrm{NaCl}, 1.5 \mathrm{mM} \mathrm{MgCl}, 0.2 \mathrm{mM}$ EDTA, $0.5 \mathrm{mM}$ DTT, 25\% glycerol, and protease inhibitor cocktail. After centrifugation at 14,000 $\mathrm{g}$ for 30 minutes, the supernatant containing the nuclear protein was removed and denatured in SDS buffer. The samples were then analyzed by immunoblot as described hereafter.

Immunoblot analysis. Retinas and cells were homogenized by sonication in the previously described immunoprecipitation (IP buffer) or RIPA buffer (for ER stress) (46). Protein concentrations were measured with the Pierce BCA reagent, and all samples were adjusted for equal protein concentration. Retinal lysates, whole-cell lysates, and nuclear extracts were analyzed using the following antibodies: $\alpha A$-crystallin (B-2, Santa Cruz) and $\alpha$ B-crystallin (ADI-SPA-223, Enzo Life Sciences), phospho-eIF2 $\alpha$ (9721S, Cell Signaling), total-eIF2 $\alpha$ (5324S, Cell Signaling), $\alpha$-tubulin (3873S, Cell Signaling), ATF4 (C-20, Cell Signaling), or CHOP (R-20, Santa Cruz). Immunoblots were performed as previously described (47) but using NuPage gels $4 \%-12 \%$ and MES buffer following the manufacturer's instructions (Thermo Fisher). Results were normalized by probing the same membrane with an antibody against $\beta$-actin (MAB-1501, Millipore), $\alpha$-tubulin (2144S, Cell Signaling), or Histone H3 (for nuclear extracts; 4499S, Cell Signaling).

Immunohistochemistry. Immunohistochemical labeling was carried out using the indirect immunofluorescence method. After being fixed for an hour in 4\% paraformaldehyde, the isolated eyecups underwent incubation in increasing concentration of sucrose solution up to $20 \%$ before being embedded and frozen in a $2: 1$ solution of $20 \%$ sucrose/OCT (optimum compound tech). Sections ( $10-\mu \mathrm{m}$ thick) at the level of the optic nerve were then obtained from each experimental group and mounted onto the same slide. Following permeabilization in $0.1 \%$ Triton X-100 for 15 minutes, the sections were incubated in a blocking solution of $1 \%$ BSA, $0.25 \%$ Triton X-100 before incubations with the primary antibodies. Double labeling was done using antibodies against $\alpha$ A-crystallin (ADI-SPA-221, Enzo Life Sciences) or $\alpha B$-crystallin (Enzo Life Sciences), along with anti-glutamine synthetase (610518, BD Biosciences), anti-GFAP (Z0334, Dako), or anti-neurofilament H (SMI-32R, Covance). Primary antibodies were detected using secondary goat anti-mouse IgG and anti-rabbit IgG antibody coupled to Alexa fluorophore 488 and 594 (Jackson Immunoresearch). Cell nuclei were counterstained using Hoechst, 1:1,000, in PBS. The same methodology was used to assess Insulin (A0564, Dako) and glucose transporter type 2 (Glut2) (Chemicon International, AB1662) in pancreas sections and BIP (ab21685, Abcam) or PDI (ab2792, Abcam) in R28 and rMC-1 cells. 
Controls were prepared by omitting the primary antibody during the incubation; in these controls, no specific staining could be detected. Images were obtained on a Leica SP5 confocal microscope and analyzed using ImageJ (NIH) software. For quantification purposes, the corrected total cell fluorescence (CTCF) was calculated by dividing the integrated density of each area by the product of total area multiplied with the background fluorescence. For each condition, the fluorescence intensities for 6 images, representative of 2 independent experiments, were summed.

Mass spectrometry analysis. For immunoprecipitation, protein G-sepharose beads (GE Healthcare) were incubated with the same $\alpha$ A-crystallin antibody used for immunoblot (Santa Cruz) and $400 \mu \mathrm{g}$ total retinal lysates (prepared as for immunoblotting). One immunoprecipitation set was used to perform immunoblots to check the efficiency of the immunoprecipitation and line up with a second SDS-PAGE gel that was fixed and stained using SYPRO Ruby (Molecular Probes) according to the manufacturer's instructions. The 2 major bands identified by Western blots were matched on the stained gel and excised under UV light.

In-gel digestion was carried out according to standard methods (48). Briefly, gel bands were dehydrated with $50 \%$ acetonitrile in $50 \mathrm{mM}$ ammonium bicarbonate buffer $(\mathrm{pH}=8.0)$. Samples were then successively incubated in DTT in $50 \mathrm{mM}$ ammonium bicarbonate and $55 \mathrm{mM}$ chloroacetamide. Gel pieces were washed twice with $50 \%$ acetonitrile in $50 \mathrm{mM}$ ammonium bicarbonate and then dried in a speed vac. Tryp$\sin (\sim 20 \mu \mathrm{l}$ of $10 \mathrm{ng} / \mu \mathrm{l}$ in ammonium bicarbonate) (Thermo Scientific Pierce) was added, and gel pieces were rehydrated overnight at $37^{\circ} \mathrm{C}$. After addition of two volumes of $50 \%$ acetonitrile in $0.5 \%$ formic acid, the supernatant was collected and dried in a speed vac.

Dried samples were dissolved in $10 \mu \mathrm{l}$ of $0.1 \%$ formic acid containing 4 unrelated reference peptides for quality control purposes, and $3 \mu 1$ were injected onto a custom-packed 5-cm C18 trapping column (Phenomenex, Luna $5 \mu \mathrm{m}, 300$ angstrom) and then resolved using a C18 (Phenomenex, Luna $3 \mu \mathrm{m}, 300$ angstrom, capillary column $100 \mu \mathrm{m}$ i.d. $\times 25 \mathrm{~cm}$ ). A nanoAquity (Waters) HLPC was used to gradient elute peptides into a triple quadrupole mass spectrometer (Thermo Scientific Vantage). Because of the number of transitions to be monitored, 2 multiple reaction monitoring experiments ( 49 transitions in the first and 56 in the second) were used to detect peptides (see Supplemental Table 1) throughout the course of the gradient elution. Specific transitions were chosen based on pilot nontargeted LC-MS/MS analysis of $\alpha$-crystallin tryptic digests performed on a Velos linear ion trap instrument (Thermo Scientific). A precursor ion window of $0.2 \mathrm{~m} / z$ and a dwell time of 50 milliseconds were used. Predicted collision energy values, data analysis, and overall peptide quantification were accomplished using Skyline (49).

Quantitative real-time PCR. Total RNA was purified with an RNA preparation kit (RNeasy Plus Mini Kit, Qiagen) and a homogenizer (QIAshredder, Qiagen). Quantitative real-time PCR and duplex qPCRs were performed as previously described (50) using gene-specific primers and fluorescent dye-labeled probes (Applied Biosystems Life Technologies). Primer-probe assay information and gene information are provided in Supplemental Table 2. Reactions were performed and monitored using a real-time PCR system (CFX384; Bio-Rad). Relative normalized mRNA levels were calculated using the $\mathrm{DDC}_{\mathrm{t}}$ method.

Proteinase K susceptibility assay. Media of $\alpha \mathrm{A}$-crystallin WT transfected $\mathrm{rMC1}$ cells, which were kept in serum-free media for 24 hours, were filtered ( $0.22 \mu \mathrm{m}$ MillexGP, Millipore) and centrifuged sequentially at $300 \mathrm{~g}$ for 6 minutes, 3,000 $\mathrm{g}$ for 20 minutes, and 5,000 $\mathrm{g}$ for 10 minutes (51). The media were concentrated using $3 \mathrm{~K}$ MWCO concentrators (Millipore). Assessment of proteinase $\mathrm{K}$ digestion of $\alpha \mathrm{A}$-crystallin in concentrated media was performed as previously described (52). $125 \mu 1$ of concentrated media were treated with $100 \mathrm{ng}$ proteinase $\mathrm{K}$ (MilliporeSigma) in a reaction containing $10 \mathrm{mM}$ Tris- $\mathrm{HCl}$ ( $\mathrm{pH}$ 7.4) with or without Triton X-100 (1\%) for 30 minutes at $37^{\circ} \mathrm{C}$. The reaction was stopped by the addition of loading buffer and heated at $70^{\circ} \mathrm{C}$ for 10 minutes. A control sample was treated the same way, aside from the omission of the enzymes. Protein levels were then assessed by immunoblotting as described above.

Conditioned media assays. Media of transfected $\mathrm{rMC} 1$ cells, which were kept in serum-free media for 24 hours, were filtered $(0.22 \mu \mathrm{m}$ MillexGP, Millipore) and centrifuged sequentially at $300 \mathrm{~g}$ for 6 minutes, 3,000 $\mathrm{g}$ for 20 minutes, and 5,000 $\mathrm{g}$ for 10 minutes (51). The media were concentrated using 3 K MWCO concentrators (Millipore). Afterward, $100 \mu 1$ concentrated media, with or without addition of an $\alpha$ A-crystallin antibody (Santa Cruz) at $1 \mu \mathrm{g} /$ well, was added to differentiated R2 8 cells (seeded at 100,000 cells per well in 96-well plates). After 4 hours of incubation, R28 cells were processed for the cell fragmentation ELISA as aforementioned.

Statistics. For all immunoblot experiments, the data were normalized to the $\beta$-actin signal as a control before analysis. ANOVA models with heterogeneous variances, adjusted for the replication of the 
experiment, were fit to the data to assess differences between diabetic and control mice. The mean \pm SEM and statistically significant differences are reported. Analyses were performed using nonrepeated-measures ANOVA, followed by the Student-Newman-Keuls test for multiple comparisons or 2-tailed $t$ test for a single comparison. A $P$ value less than 0.05 was considered significant.

Study approval. All experiments were conducted in accordance with the Association for Research in Vision and Ophthalmology Resolution on the Care and Use of Laboratory Animals and approved by the Institutional Animal Care and Use Committee of the University of Michigan.

\section{Author contributions}

AR collected and analyzed data and wrote the manuscript. JED, AMM, DS, and KMG collected and analyzed data and edited the manuscript. NWK and KS aided with data analysis and edited the manuscript. $\mathrm{PEF}$ obtained funding, designed the experiments, analyzed data, and wrote the manuscript.

\section{Acknowledgments}

This project and its authors were supported by an NEI T32-EY013934 training grant and an International Retina Research Foundation postdoctoral fellowship (to JED), a fellowship from the Dr. Werner Jackstaedt Foundation and Deutsche Forschungsgemeinschaft (RU-2020/2-1) (to AR), a Grant-in-Aid from Fight for Sight, and NIH grant EY020895 (to PEF). This work utilized the Core Center for Vision Research, funded by P30 EY007003 from the NEI. PEF is the guarantor of this work. The authors thank Eric Wawrousek for providing the $\mathrm{KO}$ mice.

Address correspondence to: Patrice E. Fort, 1000 Wall Street. Ann Arbor, Michigan 48105, USA. Phone: 734.232.8225; Email: patricef@umich.edu.

1. Klein R, Klein BE. The prevalence of age-related eye diseases and visual impairment in aging: current estimates. Invest Ophthalmol Vis Sci. 2013;54(14):ORSF5-ORSF13.

2. Amoaku WM, Saker S, Stewart EA. A review of therapies for diabetic macular oedema and rationale for combination therapy. Eye (Lond). 2015;29(9):1115-1130.

3. Barber AJ, Lieth E, Khin SA, Antonetti DA, Buchanan AG, Gardner TW. Neural apoptosis in the retina during experimental and human diabetes. Early onset and effect of insulin. J Clin Invest. 1998;102(4):783-791.

4. Martin PM, Roon P, Van Ells TK, Ganapathy V, Smith SB. Death of retinal neurons in streptozotocin-induced diabetic mice. Invest Ophthalmol Vis Sci. 2004;45(9):3330-3336.

5. Sohn EH, et al. Retinal neurodegeneration may precede microvascular changes characteristic of diabetic retinopathy in diabetes mellitus. Proc Natl Acad Sci USA. 2016;113(19):E2655-E2664.

6. Fort PE, Freeman WM, Losiewicz MK, Singh RS, Gardner TW. The retinal proteome in experimental diabetic retinopathy: up-regulation of crystallins and reversal by systemic and periocular insulin. Mol Cell Proteomics. 2009;8(4):767-779.

7. Kumar PA, Haseeb A, Suryanarayana P, Ehtesham NZ, Reddy GB. Elevated expression of alphaA- and alphaB-crystallins in streptozotocin-induced diabetic rat. Arch Biochem Biophys. 2005;444(2):77-83.

8. Brownell SE, Becker RA, Steinman L. The protective and therapeutic function of small heat shock proteins in neurological diseases. Front Immunol. 2012;3:74

9. Lim EF, et al. AlphaB-crystallin regulates remyelination after peripheral nerve injury. Proc Natl Acad Sci USA. 2017;114(9):E1707-E1716.

10. Sinha D, et al. betaA3/A1-crystallin in astroglial cells regulates retinal vascular remodeling during development. Mol Cell Neurosci. 2008;37(1):85-95.

11. Kase S, et al. alphaB-crystallin regulation of angiogenesis by modulation of VEGF. Blood. 2010;115(16):3398-3406.

12. Arac A, et al. Systemic augmentation of alphaB-crystallin provides therapeutic benefit twelve hours post-stroke onset via immune modulation. Proc Natl Acad Sci USA. 2011;108(32):13287-13292.

13. Whiston EA, et al. alphaB-crystallin protects retinal tissue during Staphylococcus aureus-induced endophthalmitis. Infect Immun. 2008;76(4):1781-1790

14. Rao NA, Saraswathy S, Pararajasegaram G, Bhat SP. Small heat shock protein $\alpha$ A-crystallin prevents photoreceptor degeneration in experimental autoimmune uveitis. PLoS ONE. 2012;7(3):e33582

15. Rao NA, Saraswathy S, Wu GS, Katselis GS, Wawrousek EF, Bhat S. Elevated retina-specific expression of the small heat shock protein, alphaA-crystallin, is associated with photoreceptor protection in experimental uveitis. Invest Ophthalmol Vis Sci. 2008;49(3):1161-1171.

16. Yaung J, Kannan R, Wawrousek EF, Spee C, Sreekumar PG, Hinton DR. Exacerbation of retinal degeneration in the absence of alpha crystallins in an in vivo model of chemically induced hypoxia. Exp Eye Res. 2008;86(2):355-365.

17. Losiewicz MK, Fort PE. Diabetes impairs the neuroprotective properties of retinal alpha-crystallins. Invest Ophthalmol Vis Sci. 2011;52(9):5034-5042.

18. Dong Z, et al. Alphab-crystallin expression in epiretinal membrane of human proliferative diabetic retinopathy. Retina (Philadelphia, Pa). 2012;32(6):1190-1196. 
19. Kase S, Ishida S, Rao NA. Increased expression of $\alpha$ A-crystallin in human diabetic eye. Int J Mol Med. 2011;28(4):505-511.

20. Schmidt T, Fischer D, Andreadaki A, Bartelt-Kirbach B, Golenhofen N. Induction and phosphorylation of the small heat shock proteins HspB1/Hsp25 and HspB5/ $\alpha$ B-crystallin in the rat retina upon optic nerve injury. Cell Stress Chaperones. 2016;21(1):167-178

21. Heise EA, Marozas LM, Grafton SA, Green KM, Kirwin SJ, Fort PE. Strain-independent increases of crystallin proteins in the retina of type 1 diabetic rats. PLoS One. 2013;8(12):e82520.

22. Brady JP, Garland D, Duglas-Tabor Y, Robison WG, Groome A, Wawrousek EF. Targeted disruption of the mouse alpha A-crystallin gene induces cataract and cytoplasmic inclusion bodies containing the small heat shock protein alpha B-crystallin. Proc Natl Acad Sci USA. 1997;94(3):884-889.

23. Brady JP, Garland DL, Green DE, Tamm ER, Giblin FJ, Wawrousek EF. AlphaB-crystallin in lens development and muscle integrity: a gene knockout approach. Invest Ophthalmol Vis Sci. 2001;42(12):2924-2934.

24. Sohn EH, et al. Retinal neurodegeneration may precede microvascular changes characteristic of diabetic retinopathy in diabetes mellitus. Proc Natl Acad Sci USA. 2016;113(19):E2655-E2664.

25. Hancock HA, Kraft TW. Oscillatory potential analysis ERGs of normal diabetic rats. Invest Ophthalmol Vis Sci. 200445(3):1002-1008

26. Kim YH, et al. Protein kinase C delta regulates anti-apoptotic alphaB-crystallin in the retina of type 2 diabetes. Neurobiol Dis. 2007;28(3):293-303.

27. Piri N, Kwong JM, Caprioli J. Crystallins in retinal ganglion cell survival and regeneration. Mol Neurobiol. 2013;48(3):819-828

28. Pangratz-Fuehrer S, Kaur K, Ousman SS, Steinman L, Liao YJ. Functional rescue of experimental ischemic optic neuropathy with $\alpha$ B-crystallin. Eye (Lond). 2011;25(6):809-817.

29. Klopstein A, et al. Beneficial effects of $\alpha$ B-crystallin in spinal cord contusion injury. J Neurosci. 2012;32(42):14478-14488.

30. Schaefer $\mathrm{H}$, et al. Study of posttranslational modifications in lenticular alphaA-Crystallin of mice using proteomic analysis techniques. Biochim Biophys Acta. 2006;1764(12):1948-1962.

31. Li R, Zhu Z, Reiser G. Specific phosphorylation of $\alpha$ A-crystallin is required for the $\alpha \mathrm{A}$-crystallin-induced protection of astrocytes against staurosporine and C2-ceramide toxicity. Neurochem Int. 2012;60(6):652-658.

32. Lind KR, Ball KK, Cruz NF, Dienel GA. The unfolded protein response to endoplasmic reticulum stress in cultured astrocytes and rat brain during experimental diabetes. Neurochem Int. 2013;62(5):784-795.

33. Mitra A, Basak T, Datta K, Naskar S, Sengupta S, Sarkar S. Role of $\alpha$-crystallin B as a regulatory switch in modulating cardiomyocyte apoptosis by mitochondria or endoplasmic reticulum during cardiac hypertrophy and myocardial infarction. Cell Death Dis. 2013;4:e582.

34. Gangalum RK, Atanasov IC, Zhou ZH, Bhat SP. AlphaB-crystallin is found in detergent-resistant membrane microdomains and is secreted via exosomes from human retinal pigment epithelial cells. J Biol Chem. 2011;286(5):3261-3269.

35. Sreekumar PG, et al. $\alpha \mathrm{B}$ crystallin is apically secreted within exosomes by polarized human retinal pigment epithelium and provides neuroprotection to adjacent cells. PLoS One. 2010;5(10):e12578.

36. Ousman SS, et al. Protective and therapeutic role for alphaB-crystallin in autoimmune demyelination. Nature. 2007;448(7152):474-479.

37. Antonetti DA, Barber AJ, Khin S, Lieth E, Tarbell JM, Gardner TW. Vascular permeability in experimental diabetes is associated with reduced endothelial occludin content: vascular endothelial growth factor decreases occludin in retinal endothelial cells. Penn State Retina Research Group. Diabetes. 1998;47(12):1953-1959.

38. Barber AJ, Antonetti DA, Gardner TW. Altered expression of retinal occludin and glial fibrillary acidic protein in experimental diabetes. The Penn State Retina Research Group. Invest Ophthalmol Vis Sci. 2000;41(11):3561-3568.

39. Reiter CE, et al. Characterization of insulin signaling in rat retina in vivo and ex vivo. Am J Physiol Endocrinol Metab. 2003;285(4):E763-E774

40. Zeng XX, Ng YK, Ling EA. Neuronal and microglial response in the retina of streptozotocin-induced diabetic rats. Vis Neurosci. 2000;17(3):463-471.

41. Barber AJ, et al. The Ins2Akita mouse as a model of early retinal complications in diabetes. Invest Ophthalmol Vis Sci. 2005;46(6):2210-2218

42. Thompson DA, et al. Rd9 is a naturally occurring mouse model of a common form of retinitis pigmentosa caused by mutations in RPGR-ORF15. PLoS One. 2012;7(5):e35865.

43. Seigel GM. Review: R28 retinal precursor cells: the first 20 years. Mol Vis. 2014;20:301-306.

44. Abcouwer SF, et al. Vascular permeability and apoptosis are separable processes in retinal ischemia-reperfusion injury: Effects of ischemic preconditioning, bevacizumab and etanercept. Invest Ophthalmol Vis Sci. 2010;51(11):5920-5933.

45. Sarthy VP, Brodjian SJ, Dutt K, Kennedy BN, French RP, Crabb JW. Establishment and characterization of a retinal Müller cell line. Invest Ophthalmol Vis Sci. 1998;39(1):212-216.

46. Reiter CE, et al. Diabetes reduces basal retinal insulin receptor signaling: reversal with systemic and local insulin. Diabetes. 2006;55(4):1148-1156.

47. Wu X, Reiter CE, Antonetti DA, Kimball SR, Jefferson LS, Gardner TW. Insulin promotes rat retinal neuronal cell survival in a p70S6K-dependent manner. J Biol Chem. 2004;279(10):9167-9175.

48. Shevchenko A, Tomas H, Havlis J, Olsen JV, Mann M. In-gel digestion for mass spectrometric characterization of proteins and proteomes. Nat Protoc. 2006;1(6):2856-2860.

49. MacLean B, et al. Skyline: an open source document editor for creating and analyzing targeted proteomics experiments. Bioin formatics. 2010;26(7):966-968.

50. Abcouwer SF, Lin CM, Shanmugam S, Muthusamy A, Barber AJ, Antonetti DA. Minocycline prevents retinal inflammation and vascular permeability following ischemia-reperfusion injury. J Neuroinflammation. 2013;10:149.

51. Théry C, Amigorena S, Raposo G, Clayton A. Isolation and characterization of exosomes from cell culture supernatants and biological fluids. Curr Protoc Cell Biol. 2006; Chapter 3:Unit 3.22.

52. Mor-Vaknin N, et al. The DEK nuclear autoantigen is a secreted chemotactic factor. Mol Cell Biol. 2006;26(24):9484-9496 